\title{
Aerodynamic shape optimization of non-straight small wind turbine blades
}

\author{
Xin Shen*, Hong Yang, Jinge Chen, Xiaocheng Zhu, Zhaohui Du \\ School of Mechanical Engineering, Shanghai Jiao Tong University, Shanghai 200240,
}

\section{China}

Abstract: Small wind turbines usually operate in sub-optimal wind conditions in order to satisfy the demand where it is needed. The aerodynamic performance of small horizontal axis wind turbines highly depends on the geometry. In the present study, the geometry of wind turbine blades are optimized not only in terms of the distribution of the chord and twist angle but also with 3- dimensional stacking line. As the blade with 3-dimensional stacking line is given sweep in the plan of rotation and dihedral in the plan containing the blade and rotor axis, the common used blade element momentum method can no longer provide accurate aerodynamic performance solution. A lifting surface method with free wake model is used as the aerodynamic model in the present work. The annual energy production and the starting performance are selected as optimization objective. The starting performance is evaluated based on blade element method. The optimization of the geometry of the non-straight wind turbine blades is carried out by using a micro-genetic algorithm. Results show that the wind turbine blades with properly designed 3- dimensional stacking line can increase the annual energy production and have a better starting behavior compared with 2- dimensional -optimized blade geometries.

Key words: wind turbine; optimization; 3- dimensional blade geometries; starting behavior

\section{Nomenclature}

$a \quad$ Axial induction factor $\quad \vec{V}_{\text {indv }} \quad$ Induced velocity

*Corresponding author. Tel.: +86 2134207714 ;

E-mail address:shenxin@sjtu.edu.cn (Xin Shen) 
$a^{\prime} \quad$ Tangential induction factor

c chord

$C_{n} \quad$ Normal force coefficient

$C_{t} \quad$ Tangential force coefficient

Rotational moment of inertia of the

Irotor rotor

$n \quad$ Blade number

Position vector of vortex collocation

$\vec{r}$ point

$r_{h} \quad$ Hub radius

$t \quad$ Time

$T_{\text {machi }}$ resistive torque

$T_{\text {shaft }}$ Aerodynamic torque generated

$\vec{V} \quad$ Velocity vector

$\vec{V}_{B} \quad$ The induced velocity from blade

$\vec{V}_{F W} \quad$ the induced velocity from far wake
The induced velocity from near wake

$V_{r e l} \quad$ Relative velocity to airfoil

$V_{x} \quad$ Axial velocity

$V_{\infty} \quad$ Free stream velocity

$\vec{w} \quad$ The induced velocity on the blade

$\alpha_{g} \quad$ Geometric angle of attack

$\alpha_{e} \quad$ Effective angle of attack

$\Phi \quad$ Flow angle

$\Gamma \quad$ Circulation

$\theta_{P} \quad$ Blade tip pitch angle

$\psi \quad$ Azimuth angle

$\Omega \quad$ Rotor rotational speed

$\psi_{b} \quad$ Blade azimuthal angle

$\zeta \quad$ Vortex age

\section{Abbreviations}

AEP Annual energy production

BEM Blade element momentum

COE Cost of energy

HAWT Horizontal-axis wind turbine

MOEA Multi-objective evolutionary algorithm

NURBS Non-uniform rational basis spline

NREL U.S. National Renewable Energy Laboratory 


\section{Introduction}

Most modern wind turbines are used for generating electricity for both large commercial wind turbines and small wind turbines. The basic design principles, such as maximum AEP and minimizing the cost of energy (COE), are the same for wind turbines of all sizes. On the other hand, there are operational issues that do depend on size; for example starting performance and cut-in speed, i.e. the lowest wind speed at which power can be extracted [1].

The working sites for large commercial wind turbines are usually specifically chosen to take advantage of high quality wind resources such as those that have a high average wind speed as well as small wind speed fluctuation throughout the year. On the other hand, small wind turbines are usually used as distributed power sources in individual homes, communities or by other users that cannot access an electricity grid and sometimes can only rely on wind power for generating their electricity. In such circumstances small wind turbines need to be able to work within a wider range of wind speeds. In many circumstance, small wind turbines could be much competitive with large wind turbines applying in distributed arrangement [2]. According to the performance analysis from previous similar studies Ozgener [3] and Al-Hadhrami [4], it is shown that the small wind turbines could be suitable tools for generating of electricity. Furthermore, the horizontal axis types of small wind turbines are demonstrated to be more efficient than vertical types of small wind turbines [4].

Unlike large wind turbines, small wind turbines often do not have a pitching control system meaning that it is not possible for small wind turbines to adjust blade pitch to suit wind conditions. In order to extract as much wind energy as possible, small wind turbines are often designed with a high tip-speed ratio that results in a large twist angle distribution along the blade in order to present high angles of attack when the rotor is stationary [1]. The high angle of attack along the blades, especially at the tip of the blade, makes it difficult to generate efficient torque to turn the blades. It is therefore important and difficult for small wind turbines to have both good starting behavior at low wind speeds and maximum AEP. Furthermore, there is increased interest in the study of blades with 3D stacking lines (swept 
and leaned blades) to improve aerodynamic efficiency, reduce the air-loads [5] and the noise of the rotor [6]. A wind turbine rotor blade, based on the U.S. National Renewable Energy Laboratory (NREL) $5 \mathrm{MW}$ reference turbine, is optimized for minimum cost of energy through simultaneous consideration of aerodynamics and bend-twist coupling by Vesel and McNamara[5]. The authors noted that successive decreases in cost of energy were observed with the coupling of bend-twist. A winglet for a HAWT rotor blade is optimized by using CFD, GA and ANN for a selected wind speed range by Elfarra et al. [6]. The results showed that the winglet affected the flow characteristics by making it more attached at the tip.

An experimental study of the starting behavior of small wind turbines was made by Ebert and Wood [7] with a 5m diameter, two-bladed small wind turbine. The observations showed that there are two main stages in the starting sequence of a $5 \mathrm{~kW}$ turbine. During the first stage, which was termed the 'idling' acceleration stage, the rotor accelerated slowly with large blade angles of attack. As the rotating velocity of the rotor increased the angles of attack of the blade decreased. During the second stage, the rotor accelerated rapidly to reach its operating rotating velocity.

Starting behavior was further studied by Mayer et al. [8] using a $5 \mathrm{~kW}$ small turbine platform with an adjustment of the pitching mechanism connecting the blades to the rotor hub. The research was focused on the relationship between the pitch angle of the blade and the starting behavior of the turbine. The results showed that with the increase of the pitch angle the 'idling' stage was shortened due to the decreasing of the angles of the attack of the blade.

Wright and Wood [9] studied the starting performance of a three-bladed, 2-m diameter HAWT via comparison of field tests measurements and calculations that employed a quasi-steady blade element analysis. The authors suggested that the blade near the hub provided most of the starting torque and that the torque for power production was generated near the tip of the blade.

Recently Pagnini et al. [10] tested the power production of two small-size commercial wind turbines. The authors noted that the overall energy production of the HAWT is higher than the VAWT. However the HAWT showed to be strongly affected by gust and large mean 
wind speed and direction fluctuations.

In literature there are three methods used in the analysis of the aerodynamic performance on horizontal axis wind turbines, the blade element momentum (BEM) theory, the vortex method and the computational fluid dynamics (CFD) methods.

Pourrajabian et al. [11] use BEM method as the aerodynamic model for the optimization of micro wind turbine blade with the objectives of both power coefficient and starting behavior at low wind speeds. Both starting time and the power coefficient of the rotor were included in the objective function. The results emphasized that the larger values of the chord and twist at the root part of the blades are indispensable for the better performance when the wind speed was low.

Sharifi and Nobari [12] developed a code based on BEM theory to predict the aerodynamics of wind turbine blades which can make possible the prediction of the best section pitch angle distribution for several sites. The BEM theory uses a simple two-dimensional analytical approach with analytical or empirical three-dimensional wake correction terms. This makes BEM theory unsuitable for the prediction of the aerodynamics of non-straight blades since this non-straight blade geometry violate some of the BEM 2D assumptions.

Vortex methods (such as lifting line and lifting surface method) represent the wind turbine blades as a series of bound vortices and trailing vortices. This capability provide flexibility that allows the vortex methods can handle 3D aerodynamic features or blade with non-straight geometry. The released root and tip vortices can be modeled with prescribed wake of free wake model. As the free wake methods do not require priori specification of the position of the vortex elements it is useful to predict transient loads and power output of wind turbine. A doubly fed induction generator (DFIG) based dynamic wind turbine model was developed by Liang et al. [13] to predict the dynamic behaviors of wind turbines both in mechanical and electrical parts. A lifting line method was used as the aerodynamic model.

CFD methods solve the full Navier-stokes equations using standard numerical techniques for partial differential equations such as finite difference, finite volume of finite element 
methods. Sayed et al. [14] also used CFD method to study the aerodynamics of wind turbine airfoil to find the suitable airfoil for the wind conditions in Egypt. A full scale wind turbine unsteady aerodynamic simulations including effects of wind shear, tower shadow and yaw regulation were carried out using CFD numerical method by Cai el al.[15]. Although the CFD methods can provide more precision prediction results than both BEM and vortex methods, such methods require huge amount of computation resource. The capabilities of such methods have not yet been validated sufficiently to be assigned the confidence levels that are necessary for design purpose.

There are a number of published papers which have described how to deal with optimization methods for wind turbine rotors. Selig et al. [16] combined a genetic algorithm with an inverse design method as an optimization method for stall-regulated HAWTs. The object of this study was to maximize the AEP. Fuglsang and Madsen [17] presented a numerical multi-disciplinary optimization method for the design of HAWTs. The method included multiple constraints and the object of this study was minimum COE, which was determined by the design minimizing fatigue and extreme loads and maximizing the AEP.

A new airfoil and a small 2-bladed wind turbine rotor were designed for low $\mathrm{Re}$ application by Singh et al. [18]. The wind turbine was also filed tested at a wind speed range of 3-6 m/s. In comparison with the baseline 3-bladed rotor, the new 2-bladed rotor produced more electrical power at the same freestream velocity. Goundar and Ahmed [19] designed a $10 \mathrm{~m}$ diameter, 3 bladed horizontal axis tidal current turbine using BEM method. Xfoil was used to develop the hydrofoil from the root to the tip of the blade.

Benini and Toffolo [20] described a multi-objective optimization method for the design of stall regulated HAWTs. Their results indicated that the minimization of COE required HAWTs having high AEPs but low blade loads and low blade weights.

Liu and Bose [21] developed a time-domain panel method for tidal turbine performance evaluation, design and optimization. The authors noted that the optimization in terms of pitch and shaft speed is the most important optimization and solidity along with number is also very influential in optimization. 
Kishinamiet el al. [22] used the blade element momentum (BEM) method to predict the aerodynamic performance of an experimental wind turbine and the results agreed relatively well with experimental measurements. Lanzafame and Messina [23] used a model based on BEM method as the aerodynamic model for the design of a micro wind turbine. Meta Heuristics algorithm was used by Tahani el al. [24] for the multi objective optimization of horizontal axis tidal current turbines based on BEM method.

Ashrafi [25] et al. developed an extended BEM method to obtain design parameters corresponding to both design and off-design conditions. The numerical model was extended to improve the blade off-design performance through finding the optimal pitch angle corrections for different operating conditions. It was found that the wind speed variations have a major impact on design parameters and reduces power coefficient significantly.

An evolutionary optimization algorithm was developed by Hampsey [26] with the objectives of both good starting performance and good peak power production for small wind turbines. The results showed that a properly designed blade with a small moment of inertia could improve the starting performance while still giving good peak power output. The author also suggested that employing a more accurate wake model was important.

The interest in the study of non-straight blades (swept and leaned blades) has increased over the last few years. Van Bussel [27] developed a moment theory to investigate the effects of adding winglets to wind turbine blades and carried out a large amount of experimental work to test the theory. He suggested that a downstream shift of the wake vorticity can increase power-output.

A free-wake lattice code was developed by Imamura et al. [28] to study the aerodynamic effects of winglets on wind turbines. A Goldstein vortex model was used by Chattot [29] to study the effects of sweep, lean and winglet on the aerodynamic performance of wind turbines. The results showed that backward-swept blades performed better than forward-swept blades.

Johansen et al. [30-32] use both the EllipSys3D CFD code and a lifting-line method to study the effect of different winglets on the power output of commercial wind turbines. They 
concluded that the use of winglets could reduce tip effects. They also suggested that adding downwind winglets can increase power output more than upwind winglets.

According to the current research on the aerodynamic design of the small wind turbine blades, the research perspectives cover a wide range, but the majority of design variables focus on the distribution of blade chord length and twist angle. It is demonstrated using the blades with 3D stacking line can improve the aerodynamic performance of wind turbines. In the present work, an optimization algorithm is applied with the aim of optimizing a small $600 \mathrm{~W}$ wind turbine non-straight blades with respect to both the starting performance and AEP. To achieve this goal, a micro-genetic algorithm is employed in view of the ability to capture global optima and a lifting-surface method with free-wake model is developed as the aerodynamic prediction model. The paper is organized as follows: first, a brief summary on the optimization design process is presented including the aerodynamic model, the design objectives, and the definition of blade geometry. In order to study the influence of the 3D shape on the aerodynamic performance of the wind turbine, both blades with and without $3 \mathrm{D}$ geometry are optimized using the same optimization objective. Finally, the results of the optimization are presented and discussed.

\section{Aerodynamic model}

Lifting surface method has been widely used in research and development of aircraft wings, hydrofoils, aerial and marine propellers and wind turbines. Zero thickness blade simulated by lifting surface theory has a history of over 60 years. The use of the lifting surface method for wind turbine aerodynamic performance prediction can be traced back to the late 1980s. A lifting surface method was developed by Kocurek [33] for the aerodynamic performance analysis of wind turbines in 1987. Recently Marten et al. [34] used a lifting line method combined with free wake model for the optimization of wind turbine blades.

\subsection{Lifting surface method with free wake model}

A lifting surface method is used as the blade model in order to take into account the 3D blade geometry and the 3D flow on the blade. Fig.1 presents the rotor body frame coordinates 
with the z-axis pointing upstream. The blade is divided into chord-wise and span-wise panels. In the present blade model, only one chord-wise panel is used and the blade is divided into a finite number of span-wise segments. The bound vortices are set at the $1 / 4$ chord and the control points are located at the $3 / 4$ chord at the center of each panel. The free vortices trailed from the ends of the bound vortices at each panel extend downstream. The trailed wake vortices comprise the near wake sheet and the far wake. The near wake extends from the bound vortices, and then rolls up into the far-wake.

Fig. 1 Schematic of rotor coordinates and the aerodynamic blade model

The circulation and the lift of each horseshoe segment are assumed to be constant. However, the adjacent segments may have unequal loadings and may therefore have different circulation strength. The circulation strength of each horseshoe filament is determined by satisfying the boundary condition on the control point at each panel. The flow on each control point is influenced by the bound vorticity of each segment and all the vortices trailed behind the blade. The induced velocity at the control point by the vortices is calculated by means of the Biot-Savart law. The boundary condition implies that the incident velocity on the blade section which is normal to the span-wise segment at the control point should be zero to prevent the flow from penetrating the blade:

$$
\vec{V}_{\infty} \cdot n_{i}+\vec{w}_{i} \cdot n_{i}=0, \quad i=1, \ldots, N s
$$

The induced velocity at the $\mathrm{i}^{\text {th }}$ control point $\vec{w}_{i}$ is equal to the sum of the induced velocities from the bound vortices, the near wake and the far wake:

$$
\vec{w}=\vec{V}_{B}+\vec{V}_{N W}+\vec{V}_{F W}
$$

The geometric structure of the far-wake is important for the induced velocity calculation at the control point. A relaxation free wake model is used to determine the shape of the far-wake. Assuming the wind turbine is working under convectional conditions, the potential factor dominates the whole flow and the viscous factor has a small influence on the wake. The vortex line can be assumed as material lines convected through the flow field at the local velocity, and a Lagrangian fluid particle is used for the description of the motion of a point on 
the far-wake filament (see Fig 1).

$$
\frac{d \vec{r}}{d t}=\vec{V}(\vec{r})
$$

where $\vec{r}$ is the position of the control point on the far-wake(free wake). On the right hand of Eq. (3) the velocity $\vec{V}(\vec{r})$ at the control point is split into two contributions: the free stream velocity and the induced velocity. The induced velocity term is comprised of the self and mutually induced velocities in the wake as well as the bound vortex on the blade:

$$
\vec{V}=\vec{V}_{\infty}+\vec{V}_{i n d v}
$$

In the fixed coordinates of the rotor, the position of the control point on the far-wake can be defined with the blade azimuthal location, $\psi$, as the temporal coordinate and vortex age, $\zeta$, at the spatial coordinate, which allows the left hand of Eq. (3)written in terms of $\psi, \zeta$ :

$$
\frac{d \vec{r}}{d t}=\frac{\partial \vec{r}}{\partial \psi} \frac{d \psi}{d t}+\frac{\partial \vec{r}}{\partial \zeta} \frac{d \zeta}{d t}
$$

Numerical solutions of free wake methodologies have been studied over decades, including relaxation schemes Bagai [35-36], Johnson [37] and Miller [38] and time-marching schemes Bhagwat [39] and Bliss [40]. Time-marching methods can potentially present the best level of approximation to the rotor wake problem with the fewest restrictions in application. However, these methods have been found to be rather susceptible to numerical instabilities [41]. On the other hand, relaxation methods enhance the ability to control the non-physical wake instabilities through explicit enforcement of wake periodicity which was first exploited by Miller [38]. Later the same method was adopted by Johnson [37] and implemented in CAMRAD (A Comprehensive Analytical Model of Rotorcraft Aerodynamics and Dynamics). Such approaches can only be used in steady-state problems.

In the present work, the aerodynamic prediction model is used to calculate the AEP from a wind turbine at a given wind distribution. It is sufficient to apply the steady lifting surface method with a relaxation free wake model to compute the steady power curve. A five-point central difference approximation is used for both the temporal and spatial derivative.

In the relaxation approach, both the temporal $\psi$ and the spatial $\zeta$ coordinates are 
treated as spatial coordinates. Then the boundary conditions in both $\psi$ and $\zeta$ directions should be specified for the relaxation scheme. In the present work, the release point of the tip vortex is set at the tip of the blade. The release point has been shown to be released at the tip of the blade in the experimental visualization studies of wind turbine rotors $[42,43]$. The strength of the tip vortex is equal to the maximum bound vorticity over the span of the blade. The periodic boundary condition is used in the $\psi$ direction:

$$
\vec{r}(0, \zeta)=\vec{r}(2 \pi, \zeta)
$$

Following this, the geometry of the tip vortex is iterated until the convergence condition is obtained.

\subsection{Validation of the aerodynamic model}

Before the aerodynamic model can be used in the design process for prediction of the loads and power output, the effectiveness of the present aerodynamic model needs to be verified.

In order to verify the aerodynamic model, the numerical predictions obtained from the present model are compared with the experimental measurements. The measurements from a two-bladed model turbine which were performed by Haans $[42,44]$ et al. will be used as a test case. The wind turbine was placed in an open-jet tunnel at Delft University of Technology. In the present study, the wake geometry measurements in both axial and yawed flow conditions and the measured thrust coefficient under axial flow conditions are used. The wind speed at which the experiment measurements were taken was kept constant at $5.5 \mathrm{~m} / \mathrm{s}$ with free-stream turbulence intensities of 1.2. The tip-speed ratio was changed by altering the rotational speed of the turbine. A more detailed description of the experiment can be found in ref [42].

Fig.2 compares the predicted and measured rotor thrust coefficient $\left(C_{T}\right)$ for three different tip pitches of $\theta_{P}=0^{\circ}, 2^{\circ}, 4^{\circ}$. Notice that there is good agreement between the predicted and measured $C_{T}$ at low load condition (large tip pitch $\theta_{P}$ or small tip-speed ratio $\lambda$ ). At small tip pitch $\left(\theta_{P}=0^{\circ}, 2^{\circ}\right)$ there is a slightly difference between the predicted and measured $C_{T}$ as the tip-speed ratio increases. When the wind turbine is working under heavy 
loaded condition (small tip pitch or large tip-speed ratio), the wind turbine blade experiences 3D effects such as stall delay and increased values of the lift force coefficient especially at the inboard regions of blade.

Fig. 2 The comparison of the predicted and measured thrust coefficient

Fig.3 gives the comparison of the tip vortex geometry structure obtained from the experiment and calculated from the present free-wake model for $\lambda=8$ and different $\theta_{P}$ under axial inflow condition. The free wake model can capture the main character of the wake behind the rotor well. After the tip vortex released, the tip vortex expands following the downstream. As the $\theta_{P}$ decreases, the expansion of the tip vortex reduces because of the reduction of the induced velocity at the disc of the rotor plane with smaller $C_{T}$. As the load on the rotor decreases, the circulation on the blade also decreases and it has less influence in the downstream. Consequently the pitch of the tip vortex increases as the pitch angle of the blade increases which is represented well with the free wake model.

Fig. 3 The comparison of the predicted and measured tip wake structure for different $\theta_{P}, \lambda=8$

\section{Optimization design process}

The design of the wind turbine is formulated as a multi-objective optimization problem with respect to the blade geometry and operation conditions (Fig.4). For a given wind speed distribution and cut-in wind speed, the ultimate goal of the small wind turbine blade design is to obtain maximum AEP and rapid acceleration to the working rotation speed from a stationary state at cut-in wind speed. Another important point is to choose the proper variables and constraints.

Fig. 4 Flow chart of the optimization design process

In the present investigation, an improved form of genetic algorithm (GA) called micro genetic algorithm $(\mu \mathrm{GA})$ is used as the optimization algorithm. The working procedure of the $\mu \mathrm{GA}$ is briefly described in the following paragraphs.

The GA is an optimization and search technique based on the principles of genetics and natural selection. Based on the principle of Darwin's theory of evolution "survival of the fittest", the GA has been demonstrated to be one of the most efficient and popular algorithms 
for optimization process. So far GA has also been used as multi-objective evolutionary algorithm (MOEA) method for wind turbine and other turbo-machinery blade design from previous similar studies Benini [45] and Bizzarrini el al. [46]. A method for three-dimensional multi-objective optimization of a transonic rotor blade was developed and tested which was based on an evolutionary algorithm and a Navier-Stokes code by Benini [45]. The $\mu \mathrm{GA}$ was used by Bizzarrini el al. [46] for the design of wind turbine airfoil in order to improve the aerodynamic efficiency of the airfoil. GA provides a better solution with a bigger population size. In order to prevent genetic drift, more than 30 individuals should be used for genetic algorithms [47]. However, a bigger population size requires more computational time to find the optimum solution. If the number of parameters is large, the length of chromosome will be big and this will necessitate a large population size. In such a situation, the traditional GA requires a huge computational effort and time [48].With the decrease of the population size, the genetic algorithm will produce poor results due to insufficient information.

The GA used in the present work is a $\mu \mathrm{GA} . \mu \mathrm{GA}$ uses a very small population sized compared to the classical GA. The small population size reduces the computational cost for each generation, which means much more generations of the $\mu \mathrm{GA}$ can be run with the same computational costs as a single generation of a classical GA. The 1st to report an implementation of a $\mu \mathrm{GA}$ was Krishnakumar [49]. Krishnakumar used a population size of 5, a crossover rate of 1 and a mutation rate of zero. The $\mu \mathrm{GA}$ has also been proved to require fewer total function evaluations than a classical GA to converge to the near-optimal region [50]. Senecal [43] have shown that the micro genetic algorithm requires a fewer number of total function evaluations compared to classical simple genetic algorithm for their test problems. The $\mu \mathrm{GA}$ has been successfully implemented as optimization algorithm for wind energy design. Dennis et al. [52] used the $\mu \mathrm{GA}$ for the optimization of turbo-machinery airfoils with the objective of minimizing the total pressure loss across the two-dimensional inlet and exit flow angles and blade cross-section area, while maintaining thickness 
distribution greater than a minimum specified value. The $\mu \mathrm{GA}$ was also used for the wind farm layout optimization in stages by DuPont and Cagan [53].

In the present work, the $\mu \mathrm{GA}$ is selected because the aerodynamics model requires a relatively large amount of CPU time for each case of simulation (about 15 seconds on the author's computer). The $\mu \mathrm{GA}$ used in the present work is the original algorithm as described by Krishnakumar [49], encoded using binary strings, the process is presented in Fig 5.

Fig. 5 The design procedure of $\mu \mathrm{GA}$

\section{Design objective}

The choice of an objective function is not always an easy task and has a significant impact on the success of an optimization process. Some earlier optimization studies of wind turbines used the minimization of cost of energy (COE) as the optimization objective function from previous studies Fuglsangand Madsen [17], Benini [20] and Xudong [54]. However the design of a wind turbine rotor is complex as design variables are dynamic and some have conflicting behaviors within the definition of the COE. For example, the rotor diameter is increased for a higher energy capture but this result into higher loads that increase the cost of energy [55].

Fuglsang and Madsen [17] used design loads of extreme forces and fatigue forces and moment as the main variables to define the cost model. Xudong et al. used the chord and mass distribution of the blades to define the cost model [54]. Other studies used a simplified function which is directly based on the aerodynamic performance of a wind turbine as the optimization objective from previous studies Selig[16], Hampsey[26] and Wood [56].

A change in the design for certain aspect of the wind turbine properties must be reflected in the cost of the production. In the present work, the objective function for the optimization is considered as:

$$
F(x)=\frac{b_{t s}+\left(1-b_{t s}\right) \bar{t}_{s}}{b_{A E P} \overline{T h r u s t}+\left(1-b_{A E P}\right) \overline{A E P}}
$$

where $b_{t s}$ and $b_{A E P}$ is the weighting coefficient which controls the contribution of each goal, AEP , the rotor thrust and the starting behavior, such that $0 \leq b_{t s}, b_{A E P} \leq 1$. 
The $\overline{t_{s}}$ and $\overline{A E P}$ are the normalized starting behavior and AEP of the optimized blade compared with the reference blade. The $\overline{\text { Thrust }}$ are the normalized thrust of the reference blade compared with the optimized blade at the average wind speed.

\subsection{Annual energy production (AEP)}

AEP is determined by the wind velocity of the wind site and the power curve of the wind turbine. In the present paper, the power curve is calculated by the lifting surface model.The AEP (assuming100\% generator efficiency) was computed based on a Rayleigh wind-speed distribution which had an average wind speed of $7.2 \mathrm{~m} / \mathrm{s}(16 \mathrm{mph})$.

The wind speed frequency distribution function is:

$$
f\left(V_{\infty}\right)=(8760 / C) k\left(V_{\infty} / C\right)^{k-1} \exp \left[-\left(V_{\infty} / C\right)^{k}\right]
$$

where $C$ is the empirical Weibull scale factor $(\mathrm{m} / \mathrm{s})$, the Weibull shape factor $k$ is set to 2 corresponding to the Rayleigh distribution. The Weibull scale factor is calculated from the average wind speed:

$$
V_{a}=C \Gamma(1+1 / k)
$$

where $V_{a}$ is the average wind speed.

The AEP is evaluated as shown in the following equation:

$$
A E P=\int_{V_{\text {in }}}^{V_{\text {out }}} p\left(V_{\infty}\right) f\left(V_{\infty}\right) d V_{\infty}
$$

where $p\left(V_{\infty}\right)$ is the wind turbine power output at wind speed $V_{\infty}$.

\subsection{Starting behavior evaluation model}

Three complex areas of aerodynamics are involved in the small wind turbine starting behavior: unsteadiness, high angles of attack, and very low Reynolds numbers. There are few references which work on the behavior of wind turbine starting. Ebert and Wood [7], Mayer et al.[8] and Wright and Wood[9] presented starting sequences from both experiments and theoretical analysis. Both reference [7] and [8] describe 30-50s 'idling' periods of slow 
rotation, with little acceleration after the initial start. In this condition the blades experience high angles of attack $(\alpha)$, are stalled, and are generating only a small amount of torque..

As mention above, there are two stages in start sequence. During the first stage, i.e. the 'idling stage', the turbine rotor rotates slowly with small acceleration and takes the main portion of the time to start. The rotational acceleration of a rotor can be expressed as:

$$
\dot{\Omega}=\frac{T_{\text {shaft }}-T_{\text {machine }}}{I_{\text {rotor }}}
$$

where $T_{\text {shaft }}$ is the aerodynamic torque generated by the rotor, $T_{\text {machine }}$ is the resistive torque caused by other components and $I_{\text {rotor }}$ is the rotational moment of inertia of the rotor which is dominated by the sum of the blades' rotational moment of inertia.

Wood [56] used a modified blade element theory to analysis starting performance and gave the aerodynamic torque contributed by a single blade element at the radius of $r$ as:

$$
T_{\text {shaft }}=N \rho V_{\infty}^{2} R^{3} \int_{\bar{r}_{h}}^{1}\left(1+\lambda^{2} r^{2}\right)^{1 / 2} \operatorname{csin} \theta_{p}\left(\cos \theta_{p}-\lambda r \sin \theta_{p}\right) r d r
$$

where $N$ is the number of blades, $\rho$ is the density of the air, $V_{\infty}$ is the wind speed, $r$ is the relative local radius, $\bar{r}_{h}$ is the relative hub radius, $\lambda$ is the tip-speed ratio, and $\theta_{p}$ is the local pitch angle(see Fig.6).

Fig. 6 Velocity triangle for a section of a blade and the local loads

The integrand in Eq.15 is a function of $\lambda, r$ and $\theta_{p}$ :

$$
F\left(\lambda, r, \theta_{p}\right)=\left(1+\lambda^{2} r^{2}\right)^{1 / 2} c \sin \theta_{p}\left(\cos \theta_{p}-\lambda r \sin \theta_{p}\right) r
$$

Differentiating $F$ with respect to $\lambda$ yields:

$$
\frac{\partial F}{\partial \lambda}=\frac{c \lambda r^{2}}{\left(1+\lambda^{2} r^{2}\right)^{\frac{1}{2}}} \sin \theta_{p}\left(\cos \theta_{p}-\lambda r \sin \theta_{p}\right) r-c\left(1+\lambda^{2} r^{2}\right)^{\frac{1}{2}} \sin ^{2} \theta_{p} r^{2}
$$

It is easily seen that $\left.\frac{\partial F}{\partial \lambda}\right|_{\lambda=0}<0$, which means that $F$ has a local maximum when $\lambda=0$.

Differentiating $F$ with respect to $\theta_{p}$ yields:

$$
\frac{\partial F}{\partial \theta_{p}}=\left(1+\lambda^{2} r^{2}\right)^{1 / 2} c\left(\cos 2 \theta_{p}-\lambda r \sin 2 \theta_{p}\right) r
$$


It can be seen that $F$ increases with $\theta_{p}$ if

$$
\theta_{p}<\frac{1}{2} \operatorname{atan} \frac{1}{\lambda r}
$$

Instead of using a time marching approach to determine the time to start [47], optimizing for better acceleration at $\lambda=0$ is also assumed improve the whole 'idling period' starting performance under the constraints of Eq.19.

When the rotor is in the 'idling period' of the starting sequence, the blade angles of attack are very large and highly separated flows occurs at the blade. It is difficult for both blade element and momentum method and the current lifting surface method to obtain accurate results under large blade angles of attack conditions.

However according to Worasinchai et al. [57] using a much more simpler modified blade element theory (BET) as the aerodynamic prediction model is also proved to be accurate to capture the measured starting performance character. In ref [57] conventional blade element momentum analysis were modified to assume no flow deceleration though the blade, and the net torque on the rotor equated to the product of rotational moment of inertia and angular acceleration. A standard 4th order Runge-Kutta method were used to determine the angular velocity as a function of time. Following Meyer and Kroger [58], Wood [59] assumed that lift and drag coefficients ( $\mathrm{Cl}$ and $\mathrm{Cd}$ respectively) for any airfoil at high a can be approximated by the 'flat plate' equations. The results in ref [59] showed that the lift and drag coefficients for $\alpha>45^{\circ}$ from 'flat plate' equations to be reasonably good for the estimating of starting performance using BET method. In the present work the same modified blade element theory is used to predict the torque at $\lambda=0$.

\section{Design variables}

It is of great importance of define the blade geometries using a parametrical modelling method during the optimization process. The basic standard for blade geometric parametrical modelling is using the least number of design variables to describe the geometry of the blade while retaining high design flexibilities.

In the present optimization process, the wind turbine blade is modelled in the form of the 
blade airfoil sections' axial distribution chord length and twist angle and the blade stacking line.

The variables used in the paper are: chord and twist distribution, the variables for blade sweep and curve description.

1. Chord distribution along the blade: A Bézier curve (Fig. 7) is used for the chord length distribution along the blade. Six control points are used to define the Bézier curve. In the present work, the maximum length is at the $25 \%$ blade station, and there is a linear length transition from the hub to the maximum chord length at the $25 \%$ blade station. The six control points are placed at the $25 \%, 40 \%, 55 \%, 70 \%, 85 \%, 100 \%$ blade stations. The same treatment of the root is used in the present work as the NREL PHASE VI rotor,i.e. the chord tapers from the maximum chord length at $25 \%$ blade span to the hub diameter of $14 \%$ blade span.

2. Twist distribution along the blade: the same method is used for the twist distribution as the chord length distribution (Fig. 7).

Fig. 7 Bézier curves for the chord and twist distribution description

3. Blade sweep and bend: swept and bent blade are usually modelled by changing the curvature of the blade stacking line in the meridional and circumferential planes. The stacking line can be defined using splines such as the Bézier spline, B-spline and NURBS [60] or elementary functions. Using splines to define the shape of the curves requires a set of control points. The shape of the spline curve can be changed by moving the position of the control point. Chattot [29] used a power law function to define the leaned shape of the blade. The blade is defined as a backward-swept and upper-bent blade. In the present work a power law function is used to describe the sweep and bend along the 
blade. The blade section is shifted to a new position according to the power law function given below:

$$
\begin{aligned}
& z_{\text {bend }}(\bar{r})=h_{l} \bar{r}^{a_{I}} \\
& y_{\text {sweep }}(\bar{r})=h_{2} \bar{r}^{a_{2}}
\end{aligned}
$$

where $h_{1}$ and $h_{2}$ (Fig. 8) are the maximum distance shifted which is the distance shifted at the tip of the blade. The changes of sweep and bend are governed by $a_{1}$ and $a_{2}$ :

$$
\bar{r}=\left(r-r_{h}\right) /\left(R-r_{h}\right)
$$

where $r_{h}$ is the radius at the hub of the blade.

Fig. 8 Schematic of the swept-curved blade

\section{Results and discussion}

In the present paper, we considered the design of a 3 bladed upwind $600 \mathrm{~W}$ small wind turbine rotor with a diameter of $1.8 \mathrm{~m}$ which operated at the rotation speed of 650 revolutions per minute (rpm). Throughout the design process, a Rayleigh wind-speed distribution with an average wind speed of $10 \mathrm{~m} / \mathrm{s}$ was used to calculate the AEP of the rotor. The cut-in wind speed was $4 \mathrm{~m} / \mathrm{s}$ and the cut out wind speed was $20 \mathrm{~m} / \mathrm{s}$.

The objectives of the present work were to design a blade set for the small wind turbine rotor with a swept and bent 3D shape. The blade geometry for the new set was to be designed for the purpose of maximum AEP minimum thrust at average wind speed and best starting behavior at the cut-in wind speed according to Eq.7.

Although many airfoils such as S822 and S834 were designed for small blades, the well-documented S809 airfoil which has extensively tested data at the wind tunnel was selected from the root to the tip of the blade for the reference and case 1 to 5 . For all the designed blades, the pitch axis was set at $30 \%$ of the chord line at the root of the blade.

\subsection{The effects of the 3D geometry shape of the blade}

The reference blade was optimized with the only objective of maximum AEP at an average wind speed of $10 \mathrm{~m} / \mathrm{s}$. In order to show the improvement of the 3D blade geometries on the aerodynamic performance of the rotor, a blade was optimized with only the chord and 
twist of the distribution (Case 1) using the same objective and then the blade with 3D blade geometries shape (Case 2) was optimized. For case 1 and case 2 the weight coefficient of $b_{t s}$ and $b_{A E P}$ of Eq.7 are both set to 0.5 .

Fig.9 shows the convergence history of the normalized objective function (Eq.7) against the numbers of generations. It is observed from fig. 8 that the case $12 \mathrm{D}$ blade has fewer design variables and thus gives faster convergence than the 3D blade in case 2 .

Fig. 9 Evolution of the normalized objective function value

Fig.10 shows the chord and twist distributions for the reference blade and the optimum blades with and without 3D blade geometries. At the inner board of the blade, the case 2 blade has the smallest chord length. As the radius increases, the case 2 blade has a larger chord length than the other two blades (95\% r/R for both the reference and case 2 blade). Both case 1 and case 2 blade have almost the same twist distribution. The reference blade has a much smaller twist distribution at the root of the blade. Both of the root twists of the blades with 2D and 3D blade geometries shown in figure 8 are nearly $60 \%$ greater than the reference blade. The twist of the case 2 blade geometries is nearly the same as the reference blade at the outer board of the blade $(r / R>0.55)$. Decreasing the chord length at the root leads to a reduction of the blade mass, which can reduce the fatigue loads of the wind turbine's main components and prolong the lifetime of the wind turbine.

Fig. 10 The chord and twist distribution of the reference blade and the optimized blades

Table 1 lists the results of the case 2 blade sweep and bend control parameters $h_{1}, h_{2}, a_{1}$ and $a_{2}$. The shape of the backward sweep and positive dihedral of the case 2 blade can be seen in Fig.11 and Fig.12.

Table 1. Results of the blade sweep and bend control parameters

\begin{tabular}{lcccc}
\hline Parameters & $h_{1}$ & $h_{2}$ & $a_{1}$ & $a_{2}$ \\
\hline results & 0.0971 & 0.0305 & 98.6518 & 3.3232 \\
\hline
\end{tabular}

Fig. 11 Sweep shapes: 2D and 3D-optimized blade

Fig. 12 Bent shapes: 2D and 3D optimized blade

It is note that for the case 2 blade geometries, the backward sweep shape of the blade changes smoothly from the root to the tip. For the bent shape the stacking line is nearly the 
same as a straight blade at most parts of the blade and evolves a sharp turning near the tip of the blade. This allows the dihedral shape of the blade to bend into a winglet smoothly at the tip.

Fig 13 shows the main results of the optimization for both blades with 2D and 3D blade geometries. The case 1 blade showed an $8.75 \%$ reduction in AEP compared with the reference blade. The reduction in energy yield is counterbalanced by a $66.9 \%$ reduction in the thrust of the rotor. The reduction in the thrust of the rotor established a reduction in the total cost and fatigue loads of the wind turbine's main components. Compared with the case 1 blade, the case 2 blade showed a 5.76\% increase in AEP while keep the thrust of rotor at the same level (with $2.2 \%$ decreasing). Both case 1 and 2 blade showed much better starting performance than the reference blade. This acceleration is the result of the low second moment of inertia of the blade and the high torque at low rotation speed. Both optimized blades have shorter chord length all along the blade than the reference blade, which give the blades with low second moment of inertia. The larger twist angle leads to high torque at a low tip-speed ratio and gives the blade better starting performance.

Fig. 13 Comparison of the optimization result

The $C_{P}$ and $C_{T}$ curve are shown in Fig.14. The optimized blades have nearly the same tendency for both $C_{P}$ and $C_{T}$ at all wind speeds. As described in the previous chapter, the AEP is the combination of the wind distribution and the power curve. In order to achieve a higher AEP, both optimized blades have a lower $C_{P \max }$ at lower wind speeds than the reference blade and the $C_{T}$ for both optimized blades is much lower than the reference blade. It is worth noting that at all wind speeds, the 3D-optimized blade has a higher $C_{P}$ and lower $C_{T}$.

Fig. 14 Power and thrust coefficient distribution of the reference and the optimized blades

The forces on the airfoil are illustrated in Fig. 15 where blade geometric angle of attack is represented as $\alpha_{g}$, the effective angle of attack is represented as $\alpha_{e}, \Phi$ is the angle between the plane of rotation and the relative velocity $V_{r e l}$ and $\theta$ is the pitch angle. For the sake of convenience from now on the angle of attack refers to the effective angle of attack.

Fig. 15 Schematic of velocity and force decompositions of a blade element

Comparisons of angle of attack distribution, tangential force and axial force provide 
insight into the discrepancies between the optimized blades. The comparison of predicted angle of attack is shown in Fig.16 for both optimized blades at a wind speed of $8 \mathrm{~m} / \mathrm{s}$. Here it can be seen that the blade with 3D blade geometries gives a lower angle of attack at the root of the blade $(\mathrm{r} / \mathrm{R}=20 \%)$ and a higher angle of attack at the outer part of blade $(\mathrm{r} / \mathrm{R}=95 \%)$. The twist angle of the case 2 blade at $20 \% \mathrm{r} / \mathrm{R}$ is higher than the case 1 blade, which is the reason why the case 2 blade has a higher angle of attack than the case 1 blade. However, at the outer part of the blade, the 3D shape alters the structure of the tip vortex which has a large influence on the induced velocities. The angle of attack, the chord length and the twist angle have a combination effect on the tangential and axial force distribution at the blade.

Fig. 16 Predicted angle of attack distribution of the optimized blades at wind speed of $8 \mathrm{~m} / \mathrm{s}$

As it can be seen from Figs.17 and 18 the blade with 3D blade geometries gives an additional driven force at the tip of the blade and can provides more torque to the main shaft because of the larger radius at the tip part of the blade. The undesired thrust force has also increased near the outer part of the blade $(85 \mathrm{r} / \mathrm{R} \sim 95 \mathrm{r} / \mathrm{R})$. However, at the tip of the blade ( $r / R>97 \%)$, the case 2 blade offers less axial force than the case 1 blade. As the radius to the tip increases, the 3D blade geometries bend towards the rotational axis. The off-axis blade tip provides less thrust to the rotor at the level of axial force than the $2 \mathrm{D}$ blade but does not have an influence on the driven force main shaft.

Fig. 17 Predicted tangential force distribution of the optimized blades at a wind speed of $8 \mathrm{~m} / \mathrm{s}$

Fig. 18 Predicted axial force distribution of the optimized blades at a wind speed of $8 \mathrm{~m} / \mathrm{s}$

\subsection{The effects of the chosen of different weigh coefficient}

The selection of weigh coefficient in Eq.7 is important for the optimization result. Although the implementation of 3D blade geometry may improve the overall aerodynamic performance of the wind turbine, it should be known that the different selection of weight coefficient value may lead to difference solutions. Three optimizations (case 3, case 4 and case 5), summarized in table 2, have been conducted with different selection of weight coefficient. All of these three cases are optimized with chord, twist distribution and 3D blade geometries. 
Table 2. Case number and associated weight coefficient included in optimization

\begin{tabular}{ccccc}
\hline & Case 2 & Case 3 & Case 4 & Case 5 \\
\hline$b_{t s}$ & 0.5 & 0.5 & 0.5 & 0.6 \\
\hline$b_{A E P}$ & 0.5 & 0.6 & 0.4 & 0.5
\end{tabular}

The final normalized performance for case 2, 3, 4 and 5 is compiled in Table 3 . The increasing of $b_{A E P}$ from 0.5 to 0.6 in case 3 compared with case 2 yields an additional AEP increasing of $0.2 \%$ as well as thrust of rotor reduction of $0.4 \%$. However the starting performance becomes worse in case 3 . The decreasing of $b_{A E P}$ from 0.5 to 0.4 in case 4 compared with case 2 yields an additional AEP reduction of $0.1 \%$ as well as worse staring performance. However case 4 demonstrates a greater leap in rotor thrust, decreasing the thrust of rotor by $1.1 \%$ from the rotor in case 2 . The increasing of $b_{t s}$ in case 5 leads to a better starting performance compared with all other cases. However the AEP and thrust of rotor in case 5 become worse, with a $0.2 \%$ reduction of AEP and $0.5 \%$ increasing of thrust of rotor.

Table 3. Normalized performance for case 2, 3 and 4 blade

\begin{tabular}{llccc}
\hline & Case 2 & Case 3 & Case 4 & Case 5 \\
\hline AEP & 1 & 1.002 & 0.999 & 0.998 \\
\hline Thrust & 1 & 0.996 & 0.989 & 1.005 \\
\hline Starting & 1 & 0.995 & 0.972 & 1.009 \\
performance & & & &
\end{tabular}

Since the starting performance is related to the blade second of inertia and the torque. Increasing the chord length and the twist angle can increase the starting torque. As it can be seen from Fig. 19, the blade in case 2 has larger chord length at the root of the blade $(\mathrm{r} / \mathrm{R}<60 \%)$. Although larger twist angle can increase the starting torque, this also shift the $C_{P \max }$ to lower wind speed and reduce the AEP of the rotor for the blade with the same chord length distribution.

Fig. 19 The chord and twist distribution of the optimized blades

Table 4 lists the results the blade sweep and bend control parameters $h_{1}, h_{2}, a_{1}$ and $a_{2}$ of the case 2, 3, 4 and 5. The blade of case 4 with the lowest level of thrust of rotor has smaller 
$a_{1}$ than the blade of case 2 and case 3 . Higher $a_{l}$ leads to sharper turning near the tip of the blade and consequently adds more power production and thrust to the rotor.

Table 4. Results of the blade sweep and bend control parameters

\begin{tabular}{lcccc}
\hline Parameters & $h_{1}$ & $h_{2}$ & $a_{1}$ & $a_{2}$ \\
\hline Case 2 & 0.0971 & 0.0305 & 98.6518 & 3.3232 \\
\hline Case 3 & 0.0982 & 0.0077 & 98.8319 & 74.1756 \\
\hline Case 4 & 0.0921 & 0.0089 & 89.1725 & 52.9438 \\
\hline Case 5 & 0.0711 & 0.0103 & 98.0513 & 4.9837
\end{tabular}

\subsection{The effects of the chosen of airfoil}

Over the past decade, commonly airfoil families such as NACA 00XX, NACA 44XX have widely been applied for wind turbines [51]. Such airfoils were designed for airplanes and working at high Re number which are significantly different from those for wind turbine airfoils. Case 6 is optimized using the airfoil NACA 0012 all alone the blade with the same weight coefficient parameters and the same wind speed distribution as well as the average wind speed as Case 2. The final normalized performance for case 2 and 6 is compiled in Table 5. The chord and twist distribution of blade in case 2 and 6 is presented in Fig.20.

Table 5. Normalized performance for case 2, 3 and 4 blade

\begin{tabular}{llll}
\hline & AEP & Thrust & Starting performance \\
\hline Case 2 & 1 & 1 & 1 \\
\hline Case 6 & 0.97 & 1.05 & 0.81 \\
\hline
\end{tabular}

Fig. 20 The chord and twist distribution of the optimized blades

The blade using NACA 0012 airfoil in Case 6 yields an AEP reduction of 3\% and an increasing the thrust of rotor of $5 \%$ as well as a worse starting performance behavior than the blade in Case 2.

Fig.21 presents the airfoil date for NACA 0012 airfoil and NREL S809 airfoil at $\operatorname{Re}=1,000,000$. As can be seen from Fig. 19 that the NACA0012 airfoil has a maximum value of $C_{l} / C_{d}$ of approximately 73 at an angle of attack $\alpha$ of $8^{\circ}$ while the NREL S809 airfoil has a maximum value of $C_{l} / C_{d}$ of approximately 125 at an angle of attack $\alpha$ of $4^{\circ}$. Higher lift:drag 
ratio of S809 air foil gives the wind turbine larger power output and larger AEP than NACA 0012 airfoil.

Fig. 21 The lift : drag ratio of NACA 0012 and NREL S809 airfoil

\section{Conclusion}

This paper has considered the optimization of small wind turbine blades with 3D blade geometries for multiple objectives, including AEP, the thrust of the rotor, and staring-ability at low wind speeds. The optimization model was based on a lifting surface method with free wake model which can take wind turbine blades with a 3D stacking line in to account. The model was validated against experimental data obtained in a two-bladed model turbine performed in an open-jet tunnel at Delft University of Technology. In the optimization, the blade geometries of both a 600W 2D and 3D wind turbine blade were optimized. The design variables used in the $2 \mathrm{D}$ blade geometry optimization were chord and twist angle and the $3 \mathrm{D}$ geometry blade optimization were chord, twist angle and the shape of the 3D stacking line for the bend and sweep shape of the blade.

The results indicate that, both the objective of maximum AEP or better starting behavior of small wind turbines have a direct impact on the geometry of the blade. The objective of maximum AEP could result in larger root chord, larger taper and smaller root twist angle. With the increase of the root chord, the rotational moment of inertia of the rotor increase and leads to worse starting behavior. The decrease of the root twist angle leads to smaller aerodynamic starting shaft torque and would also lead to worse starting behavior.

Considering the starting behavior in the objective function leads to a remarkable improving the starting behavior and a drop of decreasing of AEP. The optimized blade with 2D shape has nearly $10 \%$ of reduction in AEP and more than $66 \%$ of reduction of the rotor thrust compared with the reference blade. The results indicate that shorter chord length all along the blade gives the blades with low second moment of inertia. The larger twist angle leads to high torque at a low tip-speed ratio and gives the blade better starting performance. The blade with 3D blade geometries gives an additional driven force at the tip of the blade 
and can provides more torque to the main shaft. However the 3D blade geometries cannot enhance the starting behavior of small wind turbine efficiently at low wind speeds.

\section{Acknowledgements}

This research is supported by the National Science Foundation for Post-doctoral Scientists of China [No. 2015M571563], the National Natural Science Foundation of China [No. 51506123], the project of Shanghai Science and Technology Committee [13DZ2260900] and the 2011 Aero-Engine collaborative Innovation Plan.

\section{Reference}

[1] Wood D. Small Wind Turbines: Analysis, Design, and Application. London: Springer-Verlag, 2011.

[2] Bishop JDK, Amaratunga GAJ. Evaluation of small wind turbines in distributed arrangement as sustainable wind energy option for Barbados. Energy Conversion and Management. 2008;49(6):1652-61.

[3] Ozgener O. A small wind turbine system (SWTS) application and its performance analysis. Energy Conversion and Management. 2006;47(11-12):1326-37.

[4] Al-Hadhrami LM. Performance evaluation of small wind turbines for off grid applications in Saudi Arabia. Energy Conversion and Management. 2014;81:19-29.

[5] Vesel Jr RW, McNamara JJ. Performance enhancement and load reduction of a 5 MW wind turbine blade. Renewable Energy. 2014;66:391-401.

[6] Elfarra MA, Sezer-Uzol N, Akmandor IS. NREL VI rotor blade: numerical investigation and winglet design and optimization using CFD. Wind Energy. 2014;17(4):605-26.

[7] Ebert P, Wood D. Observations of the starting behaviour of a small horizontal axis wind turbine. Renewable energy. 1997;12(3):245-57.

[8] Mayer C, Bechly M, Hampsey M, Wood D. The starting behaviour of a small horizontal-axis wind turbine. Renewable energy. 2001;22(1):411-7.

[9] Wright AK, Wood DH. The starting and low wind speed behaviour of a small horizontal axis wind turbine. Journal of wind engineering and industrial aerodynamics. 2004;92(14-15):1265-79.

[10] Pagnini LC, Burlando M, Repetto MP. Experimental power curve of small-size wind turbines in 
turbulent urban environment. Applied Energy. 2015;154:112-21.

[11] Pourrajabian A, Ebrahimi R, Mirzaei M. Applying micro scales of horizontal axis wind turbines for operation in low wind speed regions. Energy Conversion and Management. 2014;87(0):119-27.

[12] Sharifi A, Nobari MRH. Prediction of optimum section pitch angle distribution along wind turbine blades. Energy Conversion and Management. 2013;67(0):342-50.

[13] Liang J, Qiu Y, Zhao M, Kang S, Lu H. The modeling and numerical simulations of wind turbine generation system with free vortex method and simulink. Energy Conversion and Management. 2015;103:762-77.

[14] Sayed MA, Kandil HA, Shaltot A. Aerodynamic analysis of different wind-turbine-blade profiles using finite-volume method. Energy Conversion and Management. 2012;64(0):541-50.

[15] Cai X, Gu R, Pan P, Zhu J. Unsteady aerodynamics simulation of a full-scale horizontal axis wind turbine using CFD methodology. Energy Conversion and Management. 2016;112:146-56.

[16] Selig MS, Coverstone-Carroll VL. Application of a Genetic Algorithm to Wind Turbine Design. Journal of Energy Resources Technology. 1996;118(1):22-8.

[17] Fuglsang P, Madsen HA. Optimization method for wind turbine rotors. Journal of wind engineering and industrial aerodynamics. 1999;80(1-2):191-206.

[18] Singh RK, Ahmed MR, Zullah MA, Lee Y-H. Design of a low Reynolds number airfoil for small horizontal axis wind turbines. Renewable Energy. 2012;42(0):66-76.

[19] Goundar JN, Ahmed MR. Design of a horizontal axis tidal current turbine. Applied Energy. 2013;111:161-74.

[20] Benini E, Toffolo A. Optimal Design of Horizontal-Axis Wind Turbines Using Blade-Element Theory and Evolutionary Computation. Journal of Solar Energy Engineering. 2002;124(4):357-63.

[21] Liu P, Bose N. Prototyping a series of bi-directional horizontal axis tidal turbines for optimum energy conversion. Applied Energy. 2012;99:50-66.

[22] Kishinami K, Taniguchi H, Suzuki J, Ibano H, Kazunou T, Turuhami M. Theoretical and experimental study on the aerodynamic characteristics of a horizontal axis wind turbine. Energy. 2005;30(11-12):2089-100.

[23] Lanzafame R, Messina M. Power curve control in micro wind turbine design. Energy. 2010;35(2):556-61.

[24] Tahani M, Babayan N, Astaraei FR, Moghadam A. Multi objective optimization of horizontal axis 
tidal current turbines, using Meta heuristics algorithms. Energy Conversion and Management. 2015;103:487-98.

[25] Najafian Ashrafi Z, Ghaderi M, Sedaghat A. Parametric study on off-design aerodynamic performance of a horizontal axis wind turbine blade and proposed pitch control. Energy Conversion and Management. 2015; 93: 349-356.

[26] Hampsey M. Multiobjective Evolutionary Optimisation of Small Wind Turbine Blades: Ph. D. thesis, University of Newcastle 2002.

[27] Bussel V. A momentum theory for winglets on horizontal axis wind turbine rotors and some comparison with experiments. Fourth IEA Symposium on the Aerodynamics of Wind Turbines, Rome, Italy. 1990.

[28] Imamura H, Hasegawa Y, Kikuyama K. Numerical analysis of the horizontal axis wind turbine with winglets. JSME international journal Series B, fluids and thermal engineering. 1998;41(1):170-6. [29] Chattot JJ. Effects of blade tip modifications on wind turbine performance using vortex model. Computers \& fluids. 2009;38(7):1405-10.

[30] Johansen J, Sørensen NN. Numerical analysis of winglets on wind turbine blades using CFD. 2007.

[31] Gaunaa M, Johansen J. Can CP be Increased by the Use of Winglets? A Theoretical and Numerical Investigation of the Maximum Aerodynamic Efficiency of Wind Turbine Rotors with Winglets. American Institute of Aeronautics and Astronautics, 1801 Alexander Bell Drive, Suite 500, Reston, VA, 20191-4344, USA, 2008.

[32] Johansen J, Gaunaa M, Sørensen N. Increased aerodynamic efficiency on wind turbine rotors using winglets. 26th AIAA Applied Aerodynamics Conference, Honolulu, Hawaii. 2008.

[33] Kocurek, D. Lifting surface performance analysis for horizontal axis wind turbines. No. SERI/STR-217-3163. Solar Energy Research Inst., Golden, CO (USA), 1987.

[34] Marten D, Lennie M, Pechlivanoglou G, Nayeri CN, Paschereit CO. Implementation, Optimization, and Validation of a Nonlinear Lifting Line-Free Vortex Wake Module Within the Wind Turbine Simulation Code qblade. Journal of Engineering for Gas Turbines and Power. 2016;138(7):072601.

[35] Bagai A, Leishman JG. Rotor free-wake modeling using a pseudo-implicit technique including comparisons with experimental data. Journal of the American Helicopter Society. 1995;40(3):29-41. 
[36] Bagai A, Leishman JG. Rotor free-wake modeling using a pseudoimplicit relaxation algorithm. Journal of Aircraft. 1995;32(6):1276-85.

[37] Johnson W. A General Free Wake Geometry Calculation for Wings and Rotors. Proceedings of the 51st Annual American Helicopter Society Forum, Fort Worth, Texas. 1995.

[38] Miller WO, Bliss DB. Direct periodic solutions of rotor free wake calculations. Journal of the American Helicopter Society. 1993;38(2):53-60.

[39] Bhagwat M, Leishman J. Time-accurate modeling of rotor wakes using a free-vortex wake method. 18th Applied Aerodynamics Conference: American Institute of Aeronautics and Astronautics; 2000.

[40] Quackenbush TR, Wachspress DA, Bliss DB. New free-wake analysis of rotorcraft hover performance using influence coefficients. Journal of Aircraft. 1989;26(12):1090-7.

[41] Bagai A. Contributions to the mathematical modeling of rotor flow fields using a pseudo-implicit free-wake analysis: University of Maryland, 1995.

[42] Haans W, Sant T, Van Kuik G, Van Bussel G. Measurement and modelling of tip vortex paths in the wake of a hawt under yawed flow conditions. 43rd AIAA Aerospace Sciences Meeting and Exhibit, Reno, Nevada. p. 136-45, 2005.

[43] Vermeer L, Sørensen JN, Crespo A. Wind turbine wake aerodynamics. Progress in Aerospace Sciences. 2003;39(6-7):467-510.

[44] Haans W, Sant T, van Kuik G, van Bussel G. Velocity Measurements in the Near Wake of a Horizontal Axis Wind Turbine. 31st European Rotorcraft Forum, Florence, Italy. 2005.

[45] Benini E. Three-dimensional multi-objective design optimization of a transonic compressor rotor. Journal of Propulsion and Power. 2004;20(3):559-65.

[46] Bizzarrini N, Grasso F, Coiro DP. Genetic algorithms in wind turbine airfoil design. EWEA, Brussels, Belgium. 2011.

[47] Melanie M. An introduction to genetic algorithms. Massachusetts London, England: Cambridge, 1999.

[48] Chopade RP, Mishra SC, Mahanta P, Maruyama S, Komiya A. Uniform thermal conditions on 3-D object: Optimal power estimation of panel heaters in a 3-D radiant enclosure. International Journal of Thermal Sciences. 2012;51(0):63-76.

[49] Krishnakumar K. Microgenetic algorithms for stationary and nonstationary function optimization. In SPIE Proceedings: Intelligent Control and Adaptive Systems, vol. 1196. p. 289-96, 1989. 
[50] Carroll DL. Genetic algorithms and optimizing chemical oxygen-iodine lasers. Developments in theoretical and applied mechanics. 1996;18(3):411-24.

[51] Senecal P. Development of a methodology for internal combustion engine design using multi-dimensional modeling with validation through experiments: University Microfilms International, 2000.

[52] Dennis BH, Dulikravich GS, Han Z-X. Optimization of turbomachinery airfoils with a genetic/sequential-quadratic-programming algorithm. Journal of Propulsion and Power. $2001 ; 17(5): 1123-8$.

[53] DuPont BL, Cagan J. Multi-Stage Optimization of Wind Farms with Limiting Factors. Proceedings of the ASME 2013 International Design Engineering Technical Conferences and Computers and Information in Engineering Conference, Portland, Oregon, USA. 2013.

[54] Xudong W, Shen WZ, Zhu WJ, Sørensen JN, Jin C. Shape optimization of wind turbine blades. Wind Energy. 2009;12(8):781-803.

[55] Chehouri A, Younes R, Ilinca A, Perron J. Review of performance optimization techniques applied to wind turbines. Applied Energy. 2015;142:361-88.

[56] Wood D. Dual Purpose Design of Small Wind Turbine Blades. Wind Engineering. 2004;28(5):511-27.

[57] Worasinchai S, Ingram GL, Dominy RG. Effects of Wind Turbine Starting Capability on Energy Yield. Journal of engineering for gas turbines and power. 2012;134(4).

[58] Meyer C, Kröger D. Numerical simulation of the flow field in the vicinity of an axial flow fan. International journal for numerical methods in fluids. 2001;36(8):947-69.

[59] Wood DH. A blade element estimation of the cut-in wind speed of a small turbine. Wind Engineering. 2001;25(2):125-30.

[60] He L, Shan P. Three-Dimensional Aerodynamic Optimization for Axial-Flow Compressors Based on the Inverse Design and the Aerodynamic Parameters. Journal of Turbomachinery. 2011;134(3):031004-. 


\section{LIST OF FIGURES}

Fig. 1 Schematic of rotor coordinates and the aerodynamic blade model

Fig. 2 The comparison of the predicted and measured thrust coefficient

Fig. 3 The comparison of the predicted and measured tip wake structure for different $\theta_{P}, \lambda=8$

Fig. 4 Flow chart of the optimization design process

Fig. 5 The design procedure of $\mu \mathrm{GA}$

Fig. 6 Velocity triangle for a section of a blade and the local loads

Fig. 7 Bézier curves for the chord and twist distribution description

Fig. 8 Schematic of the swept-curved blade

Fig. 9 Evolution of the normalized objective function value

Fig. 10 The chord and twist distribution of the reference blade and the optimized blades

Fig. 11 Sweep shapes: 2D and 3D-optimized blade

Fig. 12 Bent shapes: 2D and 3D optimized blade

Fig. 13 Comparison of the optimization result

Fig. 14 Power and thrust coefficient distribution of the reference and the optimized blades

Fig. 15 Schematic of velocity and force decompositions of a blade element

Fig. 16 Predicted angle of attack distribution of the optimized blades at wind speed of $8 \mathrm{~m} / \mathrm{s}$

Fig. 17 Predicted tangential force distribution of the optimized blades at a wind speed of $8 \mathrm{~m} / \mathrm{s}$

Fig. 18 Predicted axial force distribution of the optimized blades at a wind speed of $8 \mathrm{~m} / \mathrm{s}$

Fig. 19 The chord and twist distribution of the optimized blades

Fig. 20 The chord and twist distribution of the optimized blades

Fig. 21 The lift : drag ratio of NACA 0012 and NREL S809 airfoil 


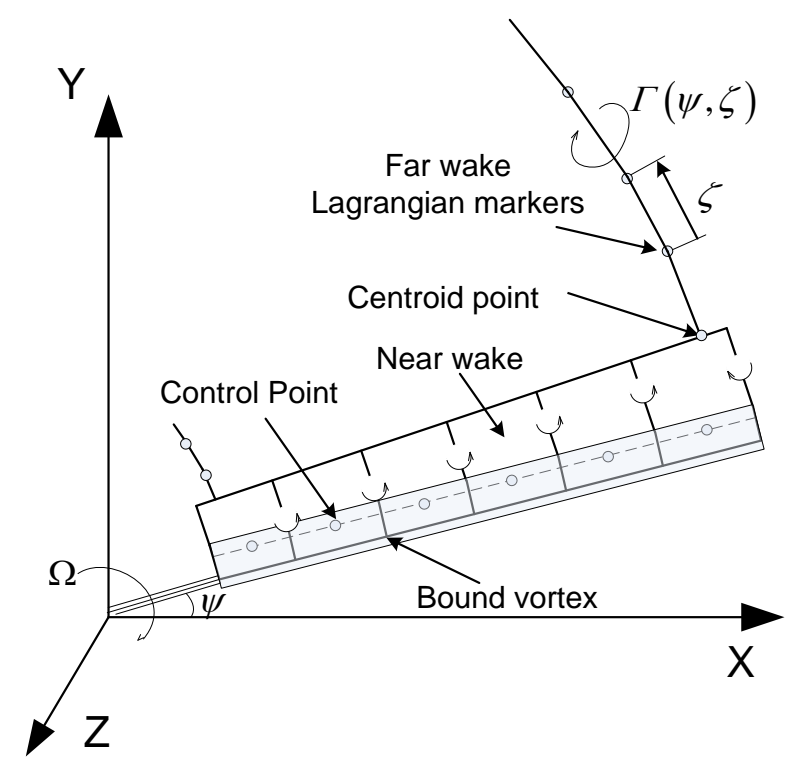

Fig. 1 Schematic of rotor coordinates and the aerodynamic blade model

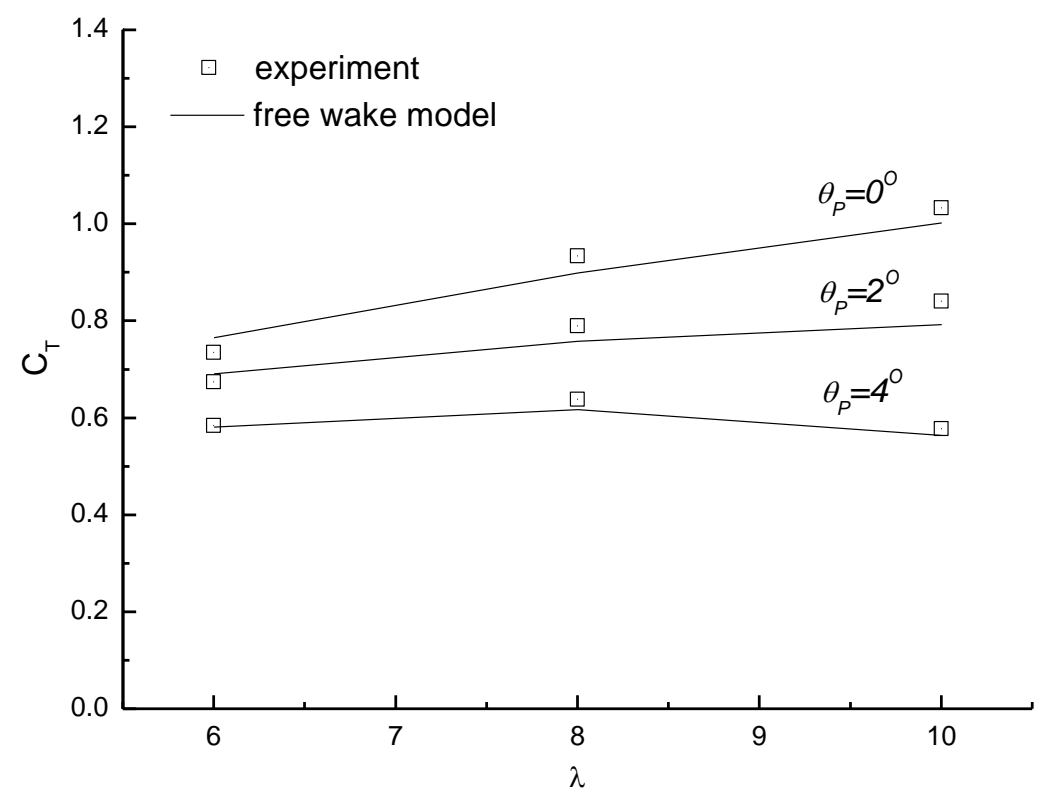

Fig. 2 The comparison of the predicted and measured thrust coefficient 


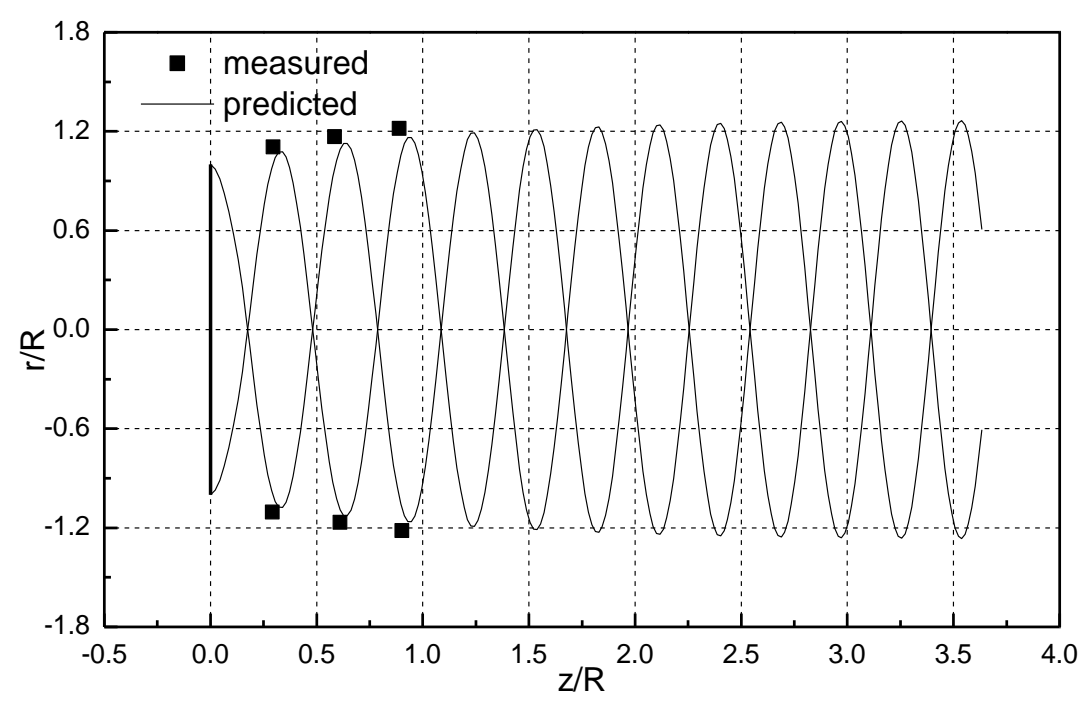

(a) $\lambda=8, \theta_{P}=0^{\circ}$

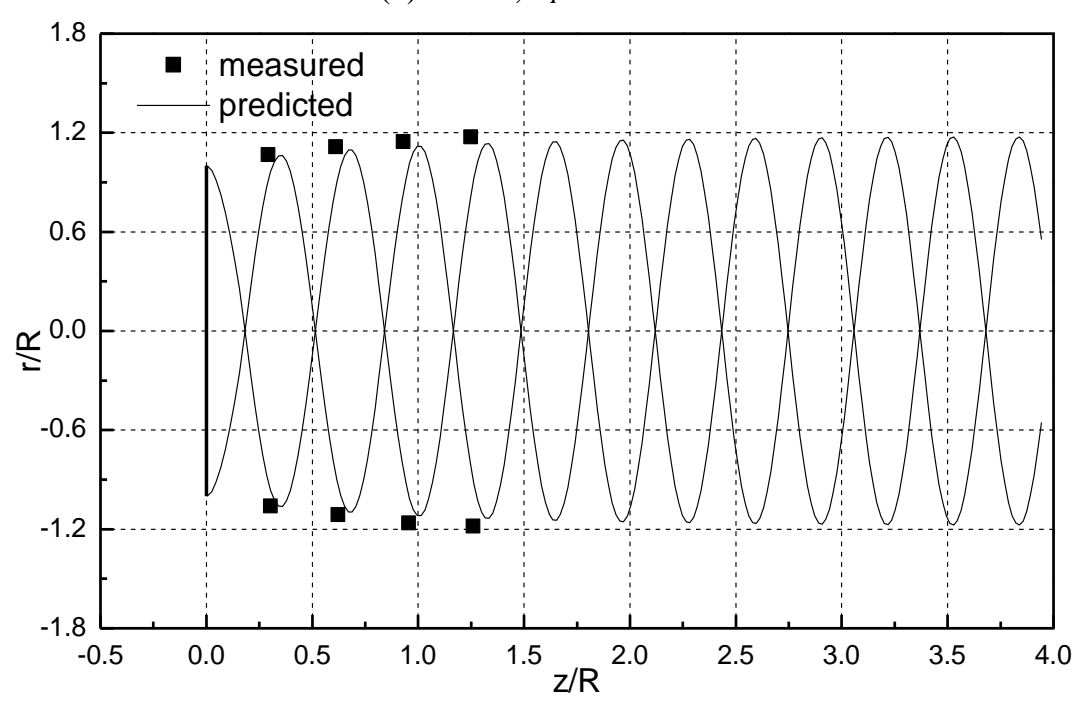

(b) $\lambda=8, \theta_{P}=2^{\circ}$

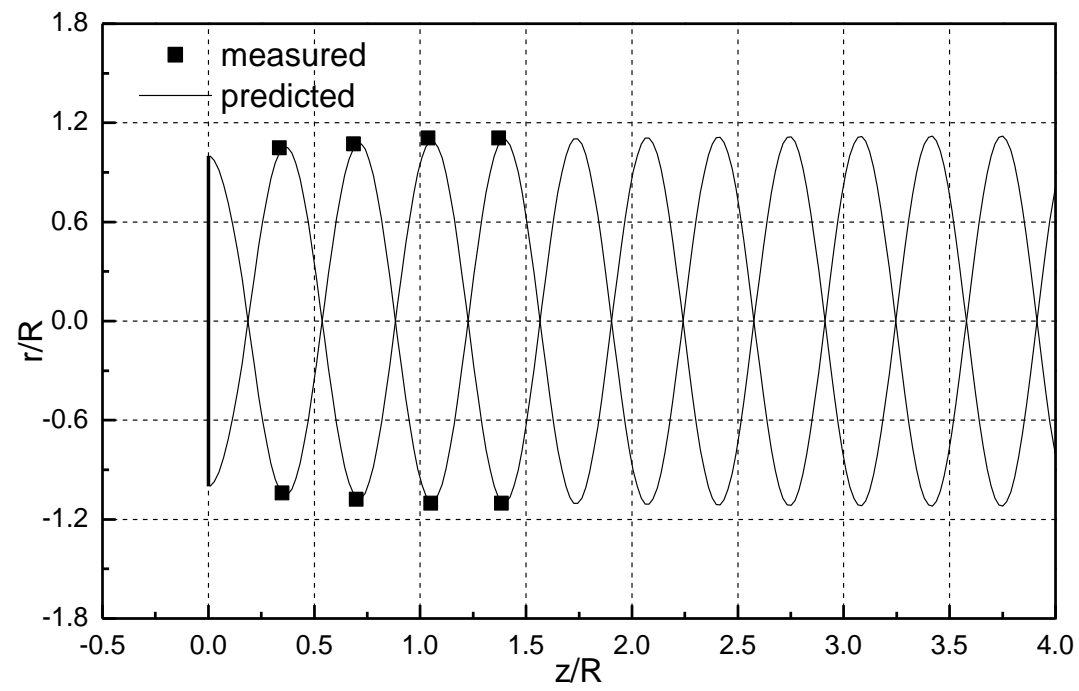


(c) $\lambda=8, \theta_{P}=4^{\circ}$

Fig. 3 The comparison of the predicted and measured tip wake structure for different $\theta_{P}, \lambda=8$

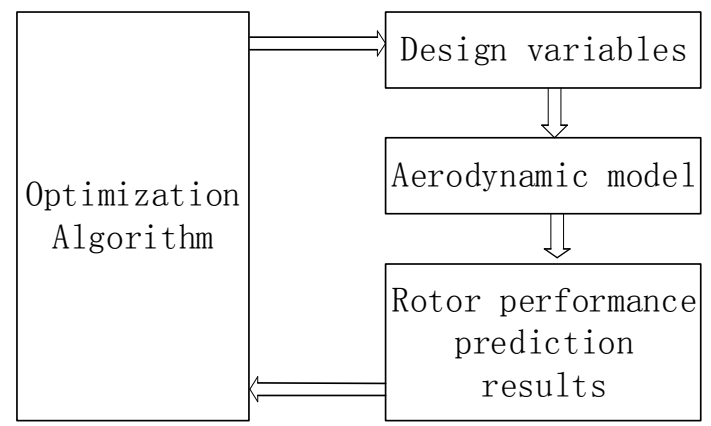

Fig. 4 Flow chart of the optimization design process

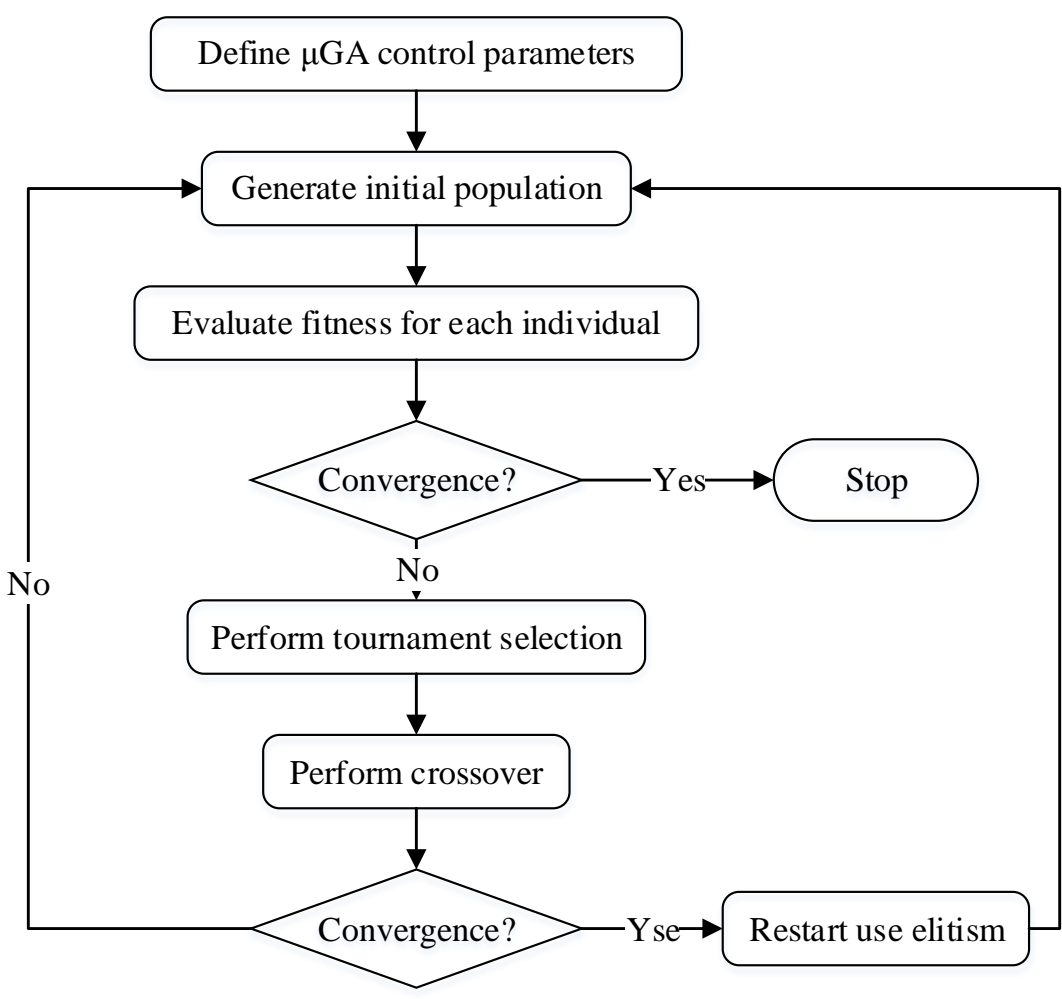

Fig. 5 The design procedure of $\mu \mathrm{GA}$

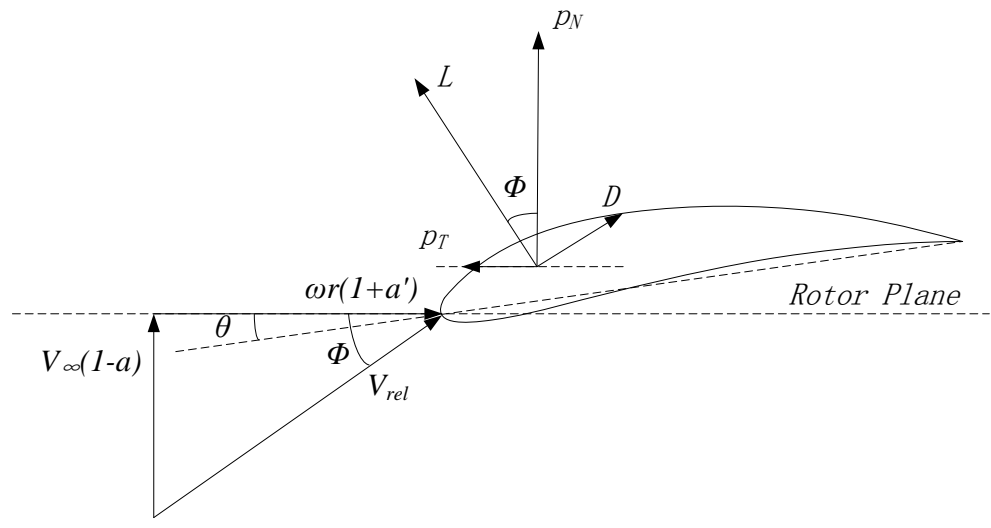


Fig. 6 Velocity triangle for a section of a blade and the local loads

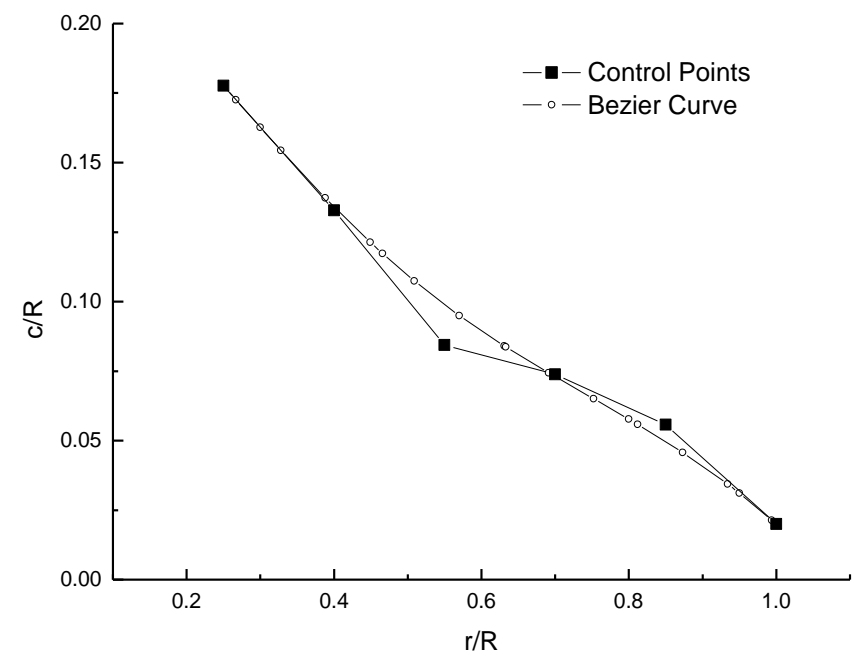

(a) Chord distribution

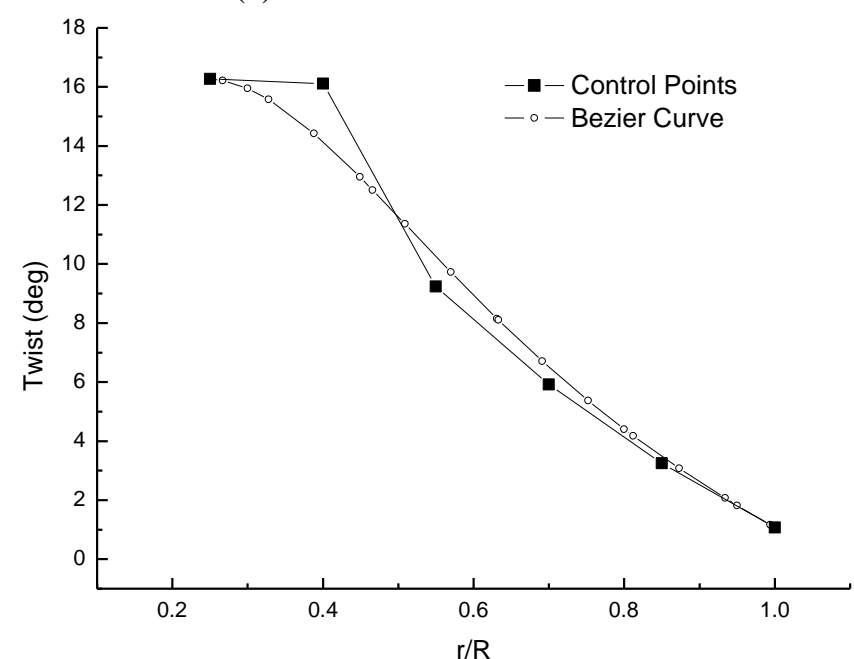

(b) Twist distribution

Fig. 7 Bézier curves for the chord and twist distribution description

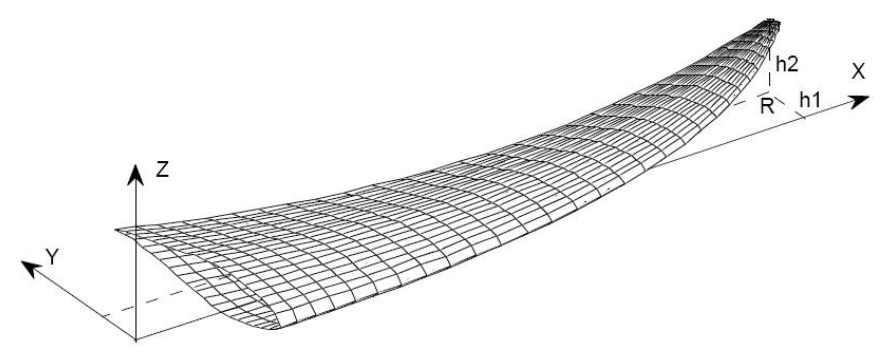

Fig. 8 Schematic of the swept-curved blade 


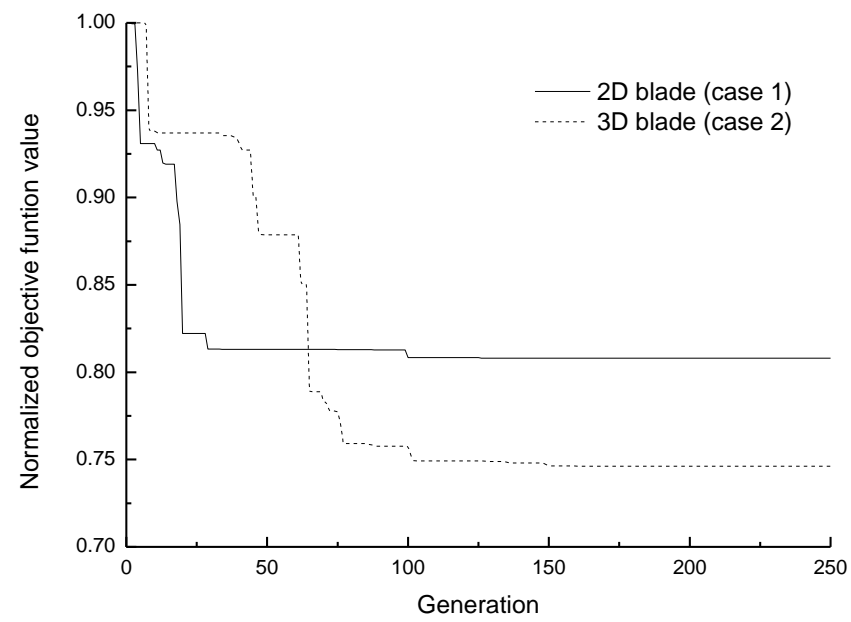

Fig. 9 Evolution of the normalized objective function value

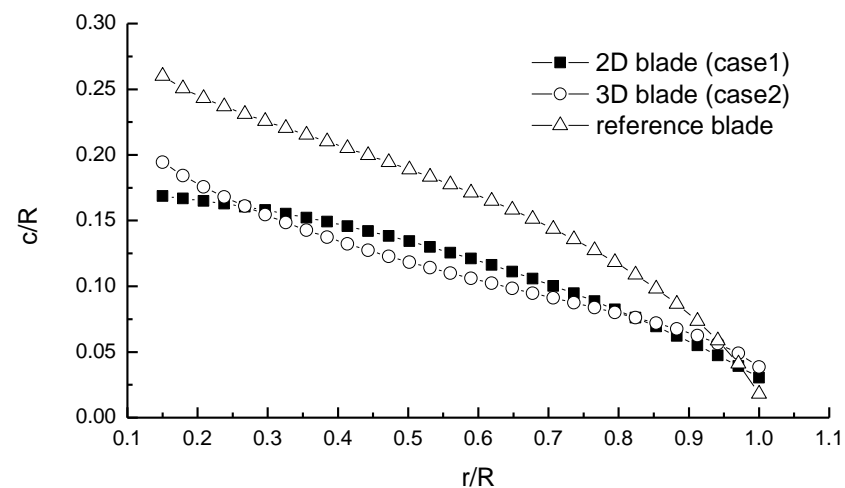

(a) Chord distribution

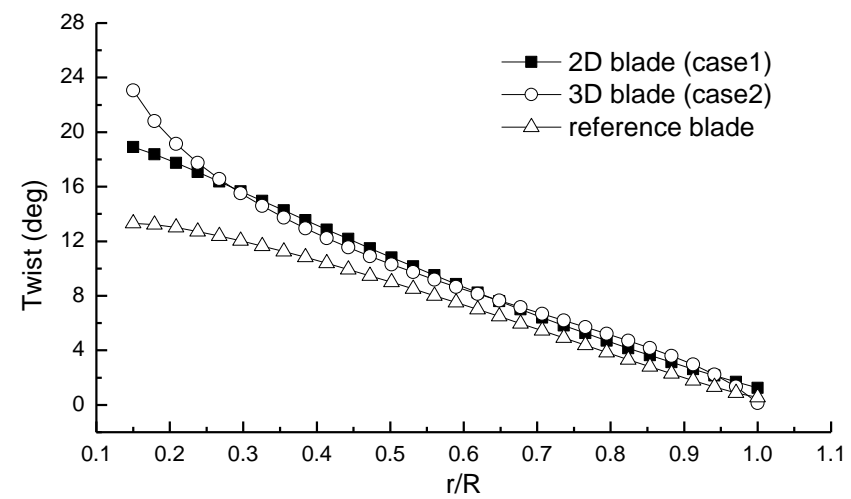

(b) Twist distribution

Fig.10 The chord and twist distribution of the reference blade and the optimized blade 


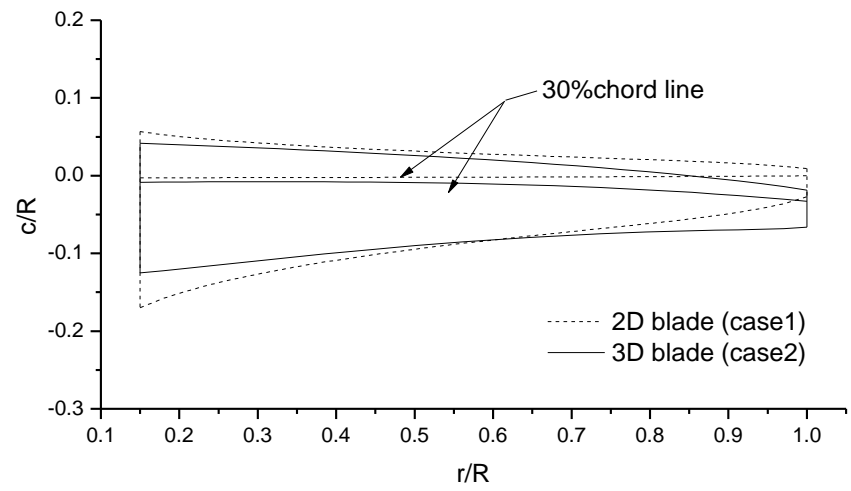

Fig.11 Sweep shapes: 2D and 3D-optimized blade

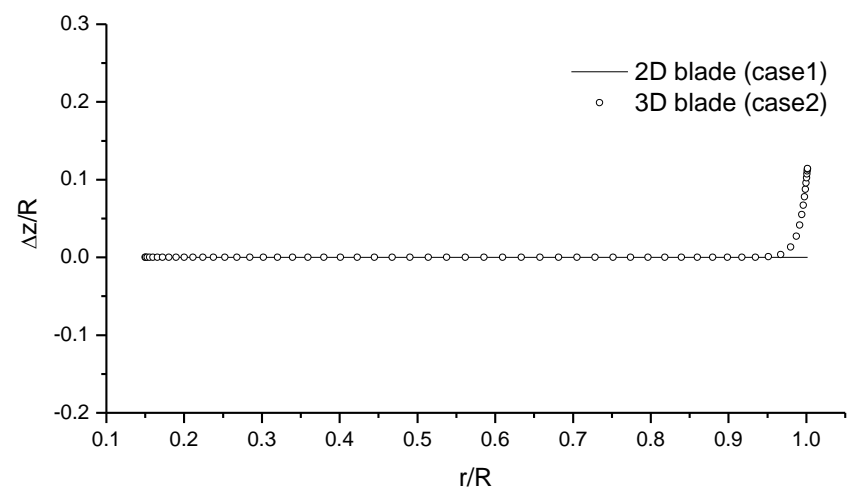

Fig.12 Bent shapes: 2D and 3D optimized blade

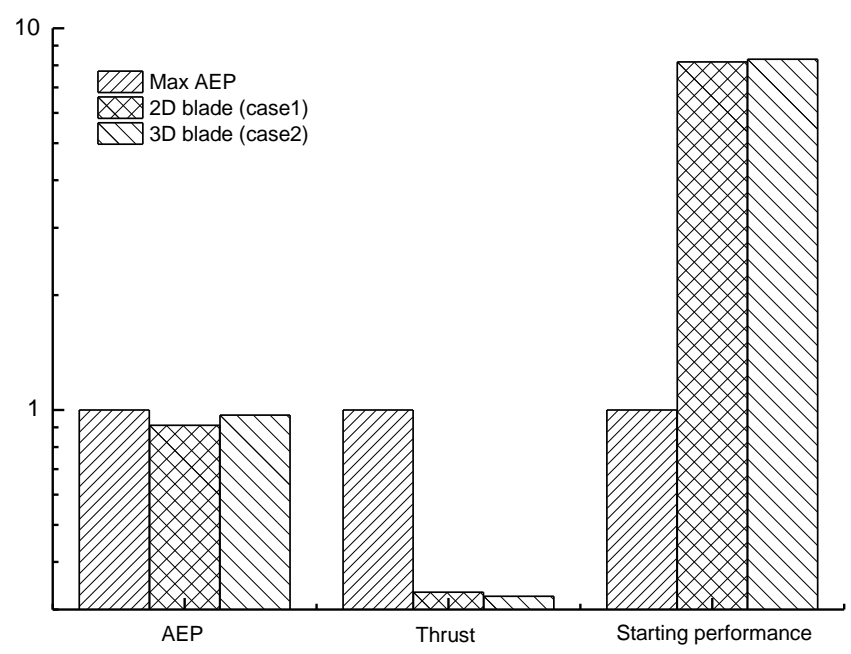

Fig.13 Comparison of the optimization result 


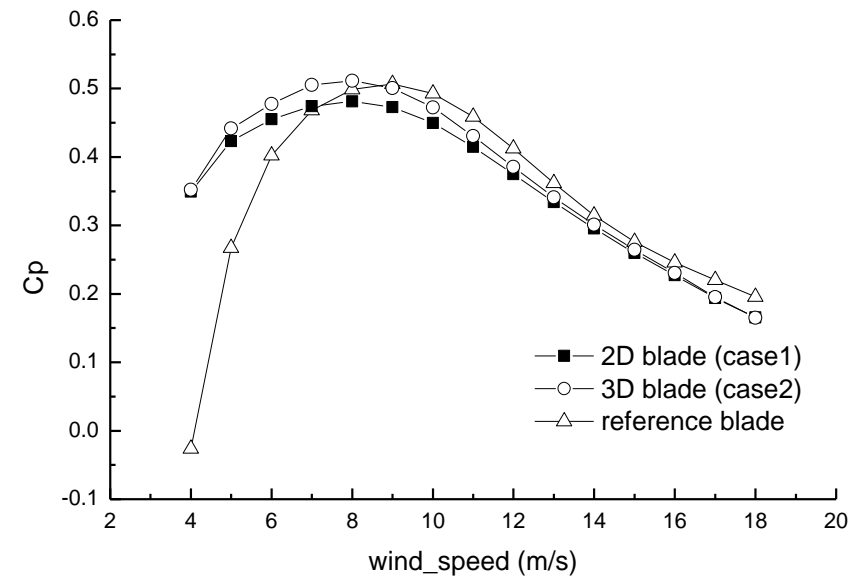

(a) Power coefficient, $C_{P}$, distribution

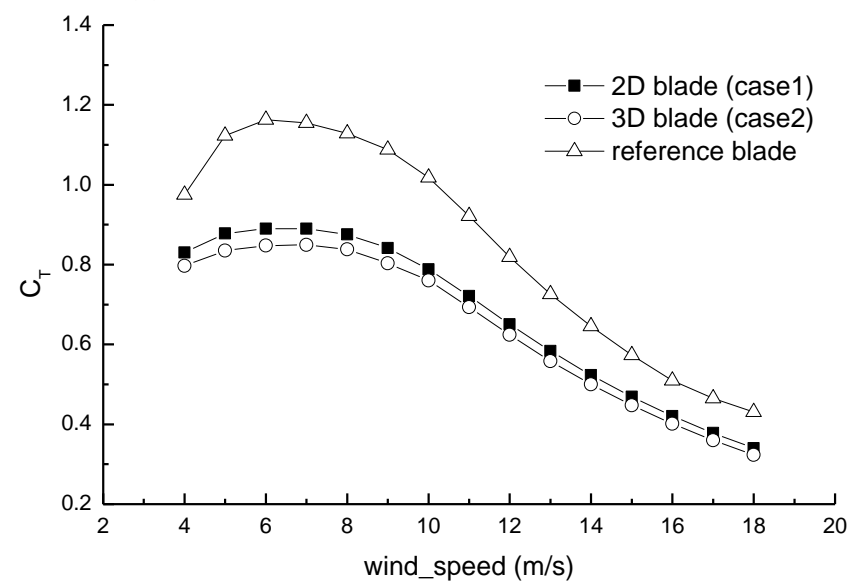

(b) Thrust coefficient, $C_{T}$, distribution

Fig.14 Power and thrust coefficient distribution of the reference

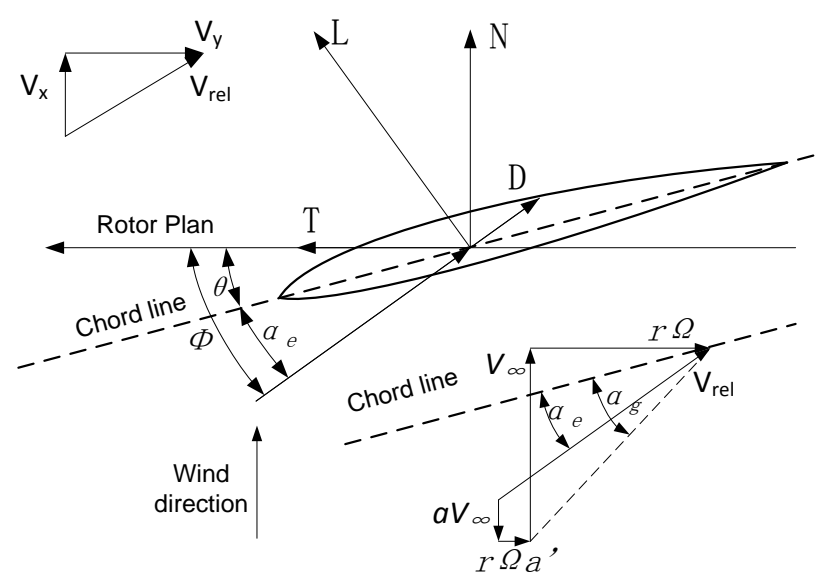

Fig.15 Schematic of velocity and force decompositions of a blade element 


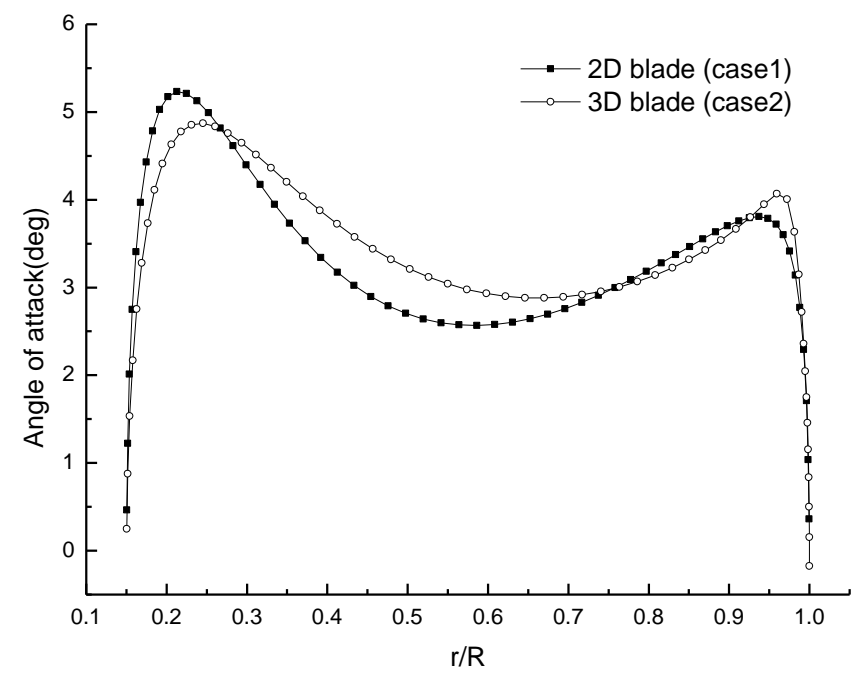

Fig.16 Predicted angle of attack distribution of the optimized blades at wind speed of $8 \mathrm{~m} / \mathrm{s}$

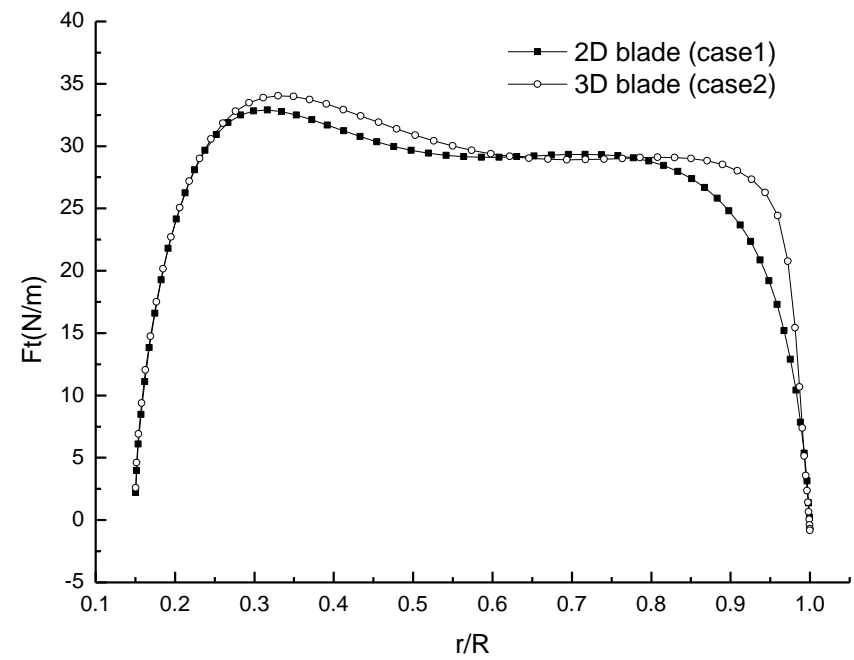

Fig.17 Predicted tangential force distribution of the optimized blades at a wind speed of $8 \mathrm{~m} / \mathrm{s}$

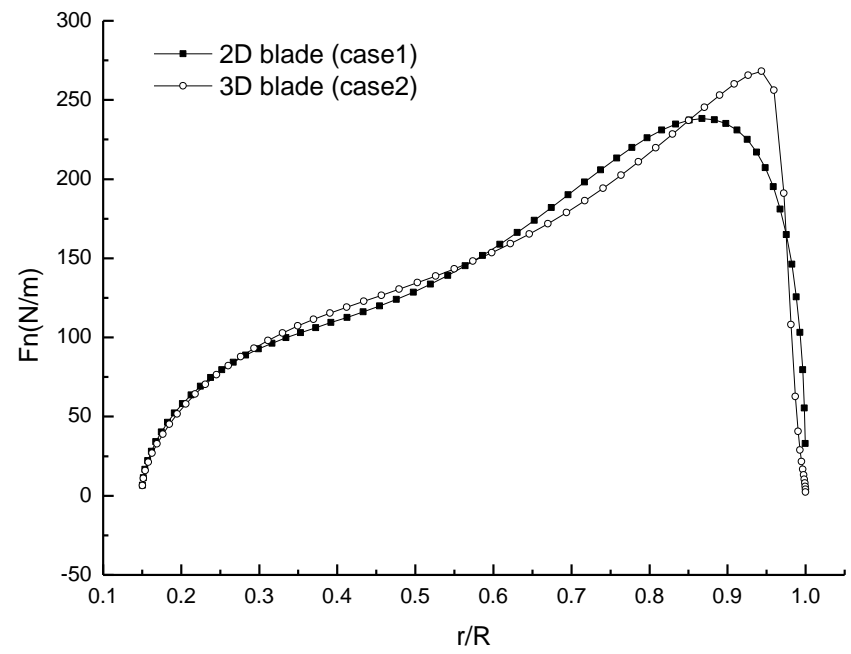

Fig.18 Predicted axial force distribution of the optimized blades at a wind speed of $8 \mathrm{~m} / \mathrm{s}$ 


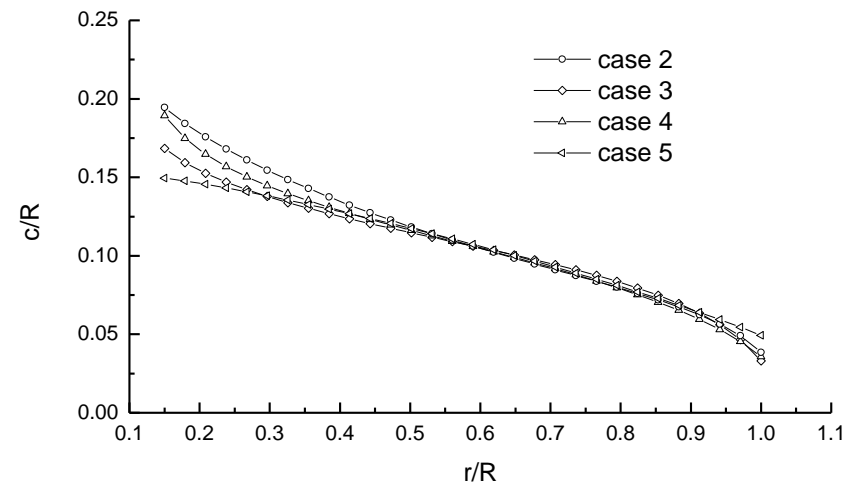

(a) Chord distribution

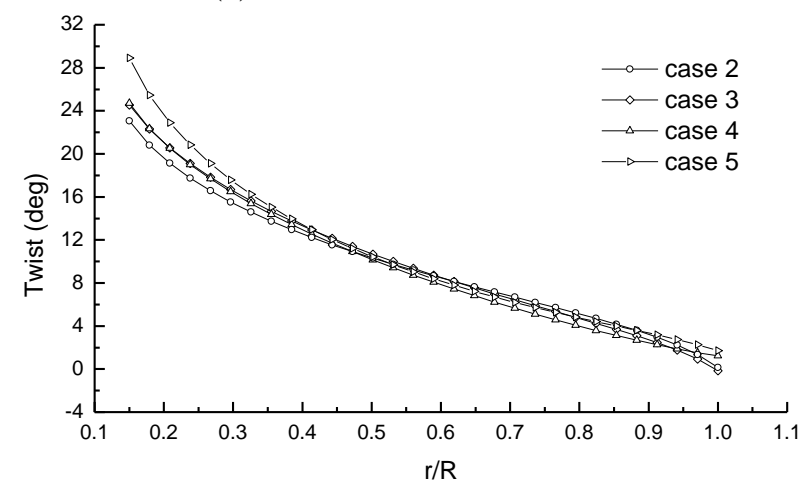

(b) Twist distribution

Fig.19 The chord and twist distribution of the optimized blades

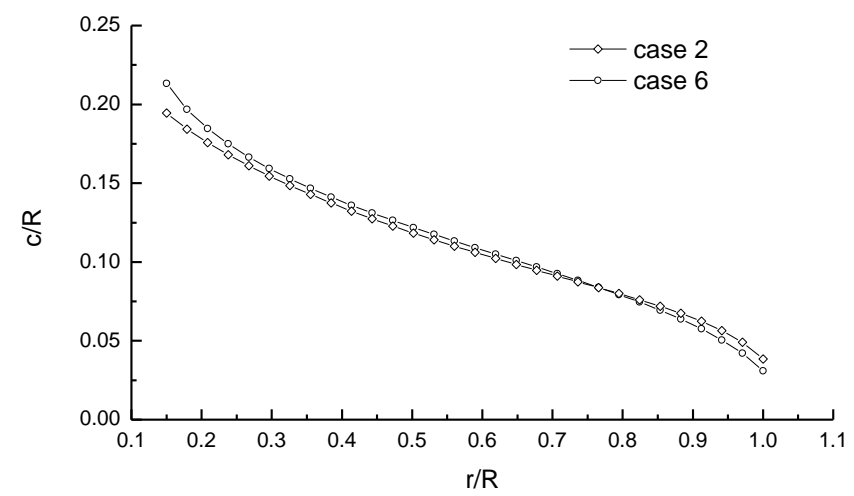

(a) Chord distribution

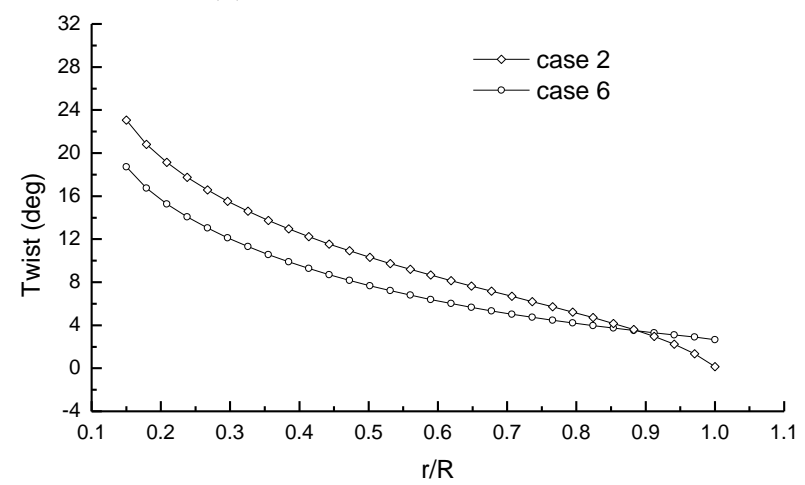

(b) Twist distribution 
Fig.20 The chord and twist distribution of the optimized blades

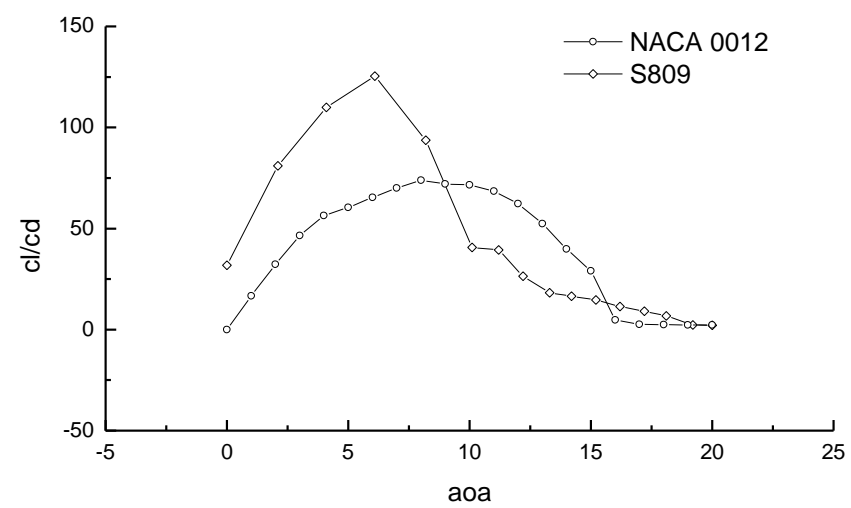

Fig.21 The lift : drag ratio of NACA 0012 and NREL S809 airfoil 


\section{Graphical abstract}

Small wind turbine blades with 3D stacking lines (sweep and bend) have been considered and analyzed with an optimization code based on the lifting surface method. The results indicated that the power capture and the rotor thrust can be improved with these more complex geometries. The starting behavior of the small wind turbines can be improved by the optimization of the blade chord and twist angle distribution.
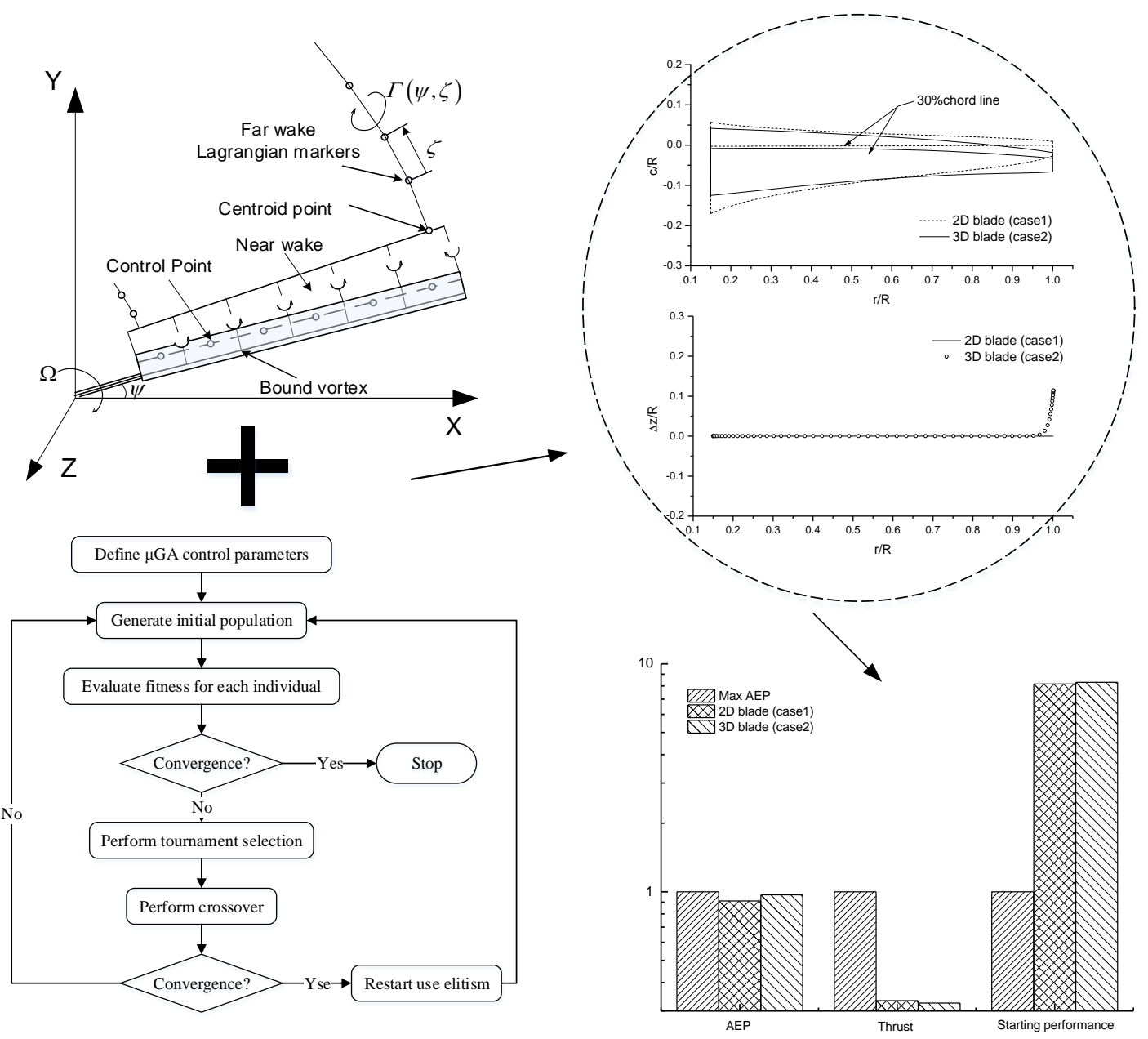